\title{
On the Convergence of the Goerisch Method for Self-Adjoint Eigenvalue Problems with Arbitrary Spectrum
}

\author{
U. Mertins
}

In remembrance of Friedrich Goerisch

\begin{abstract}
It was shown recently in [13] that the Goerisch method provides upper and lower bounds to eigenvalues of variationally posed self-adjoint eigenvalue problems with arbitrary spectrum. In the present paper the approximation of eigenelements is established. In addition, the convergence of the eigenvalue and eigenelement approximations is shown in a pure functional analytic procedure. A numerical example is given where the curve veering phenomenon occurs.

Keywords: Eigenvalue problems, variational methods, upper and lower bounds to eigenvalues, approximation of eigenelements, convergence of the Goerisch method, curve veering
\end{abstract}

AMS subject classiflcation: $49 \mathrm{R} 05,65 \mathrm{~N} 12,65 \mathrm{~N} 25,65 \mathrm{~N} 30,35 \mathrm{P} 15$

\section{Introduction}

For self-adjoint eigenvalue problems the famous Rayleigh-Ritz method allows for a straightforward and efficient computation of upper bounds to eigenvalues below the essential spectrum as well as approximations to the corresponding eigenelements. As is well known, the method converges (see, e.g., [8, 9]). Lehmann [6], Maehly [7] and Goerisch [3] developed complementary methods that provide a possibility to calculate corresponding lower bounds to the eigenvalues. From a combination of these methods very satisfying inclusion intervals of the eigenvalues are obtained (for further references see [13]). Zimmermann [12] has shown that the error in a Lehmann-Maehly-Goerisch bound is less than a constant multiple of the error in the corresponding Rayleigh-Ritz bound.

The methods mentioned are no longer available for isolated interior eigenvalues, i.e. eigenvalues lying in a gap of the essential spectrum. As shown in [13] there are two versions of Goerisch's method giving upper and lower bounds to all isolated eigenvalues of finite multiplicity. We shall derive an extension of Goerisch's method that yields approximations also to eigenelements. In addition to this, general convergence results are given for eigenvalue and eigenelement approximations attained by this method (Theorems $1.2,1.3,6.2$ and 6.3 ).

U. Mertins: Techn. Univ. Clausthal, Inst. für Math., Erzstr. 1, D - 38678 Clausthal Zellerfeld 
By establishing further insight into the methods, the proofs are in themselves of some theoretical interest. In particular, the Lehmann-Maehly method results in a special application of the Rayleigh-Ritz procedure for the calculation of eigenvalue bounds of a transformed problem (Theorems 2.6 and 7.1). In Section 5 and Section 8 a numerical example, already treated in [13], for a system of partial differential equations shows the efficiency of the evolved theory.

\section{Convergence results for the left-definite eigenvalue problem}

Let $\mathbf{H}_{a}$ be a separable, complex Hilbert space with inner product $a(\cdot, \cdot)$ and norm $\|\cdot\|_{a}$. Suppose $b(\cdot, \cdot)$ is a continuous, Hermitian, sesquilinear form on $\mathbf{H}_{\boldsymbol{a}} \times \mathbf{H}_{\boldsymbol{a}}$ such that, for $u \in \mathbf{H}_{a}, b(u, v)=0$ for all $v \in \mathbf{H}_{a}$ implies $u=0$. We then consider the following left-definite, variationally posed eigenvalue problem:

$$
\left.\begin{array}{l}
\text { Find eigenpairs }(\lambda, u) \in \mathbb{R} \times \mathbf{H}_{a} \text { with } u \neq 0 \\
\text { such that } a(u, v)=\lambda b(u, v) \text { holds for all } v \in \mathbf{H}_{\mathbf{a}} .
\end{array}\right\}
$$

Denote by $B \in \mathcal{L}\left(\mathbf{H}_{a}\right)$ the bounded self-adjoint operator that satisfies

$$
a(B u, v)=b(u, v) \quad \text { for all } u, v \in \mathbf{H}_{\mathbf{a}} .
$$

Then $B$ possesses a self-adjoint inverse

$$
A=B^{-1}: \mathbf{H}_{a} \supset \mathcal{D}(A) \longrightarrow \mathbf{H}_{a}
$$

and problem (1.1) is equivalent to the eigenvalue problem for the operator $A$. Hence, $\sigma(A)$ and $\sigma_{e}(A)$ represent the spectrum and the essential spectrum of (1.1) with $0 \notin$ $\sigma(A)$.

According to [13: Section 1], we introduce for a spectral parameter $\rho \in \mathbb{R}$ a local notation for the eigenvalues of finite.multiplicity

$$
\rho^{-}<\lambda_{-k_{\rho}^{-}}^{\rho} \leq \cdots \leq \lambda_{-2}^{\rho} \leq \lambda_{-1}^{\rho}<\rho<\lambda_{+1}^{\rho} \leq \lambda_{+2}^{\rho} \leq \cdots \leq \lambda_{+k_{\rho}^{+}}^{\rho}<\rho^{+}
$$

with $k_{\rho}^{-}, k_{\rho}^{+} \in \mathbb{N}_{0}, \rho^{-} \in \mathbb{R} \cup\{-\infty\}$ and $\rho^{+} \in \mathbb{R} \cup\{+\infty\}$. Let the eigenelements $u_{ \pm i}^{\rho} \in \mathbf{H}_{a}$ of eigenpairs. $\left(\lambda_{ \pm i}^{\rho}, u_{ \pm i}^{\rho}\right) \in \mathbb{R} \times \mathbf{H}_{a}$ satisfy:

$$
a\left(u_{k}^{\rho}, u_{l}^{\rho}\right)=\delta_{k l} \quad\left(k, l \in\left\{-k_{\rho}^{-}, \ldots,-1,+1, \ldots,+k_{\rho}^{+}\right\}\right) .
$$

We set

$$
D_{\rho}=\left\{\dot{u} \in \mathbf{H}_{a}: a(u, v)=\rho b(u, v) \text { for all } v \in \mathbf{H}_{\mathbf{a}}\right\} .
$$

In addition to these assumptions suppose that $\mathbf{X}$ is a complex Hilbert space with inner product $s(\cdot, \cdot)$ and norm $\|\cdot\|_{s}$. By the isometry $\mathbf{T}: \mathbf{H}_{\boldsymbol{a}} \longrightarrow \mathbf{X}$,

$$
s(\mathbf{T} u, \mathbf{T} v)=a(u, v) \quad \text { for all } u, v \in \mathbf{H}_{a}
$$


let $\mathbf{H}_{a}$ be embedded into $\mathbf{X}$. Denote by

$$
\mathbf{X}^{\circ}=\left\{w \in \mathbf{X}: s(w, \mathbf{T} u)=0 \text { for all } u \in \mathbf{H}_{\mathbf{a}}\right\}
$$

the $s(\cdot, \cdot)$-orthogonal complement of $\mathbf{T H}_{a}$ in $\mathbf{X}$ and by

$$
Q^{\circ}: \mathbf{X} \longrightarrow \mathbf{X}^{\circ}
$$

the $s(\cdot, \cdot)$-orthogonal projection of $\mathbf{X}$ onto $\mathbf{X}^{\circ}$. For $v \in \mathbf{H}_{a}$,

$$
\begin{aligned}
\min \left\{s(w, w): w \in \mathbf{T} B v+\mathbf{X}^{\circ}\right\} & =s\left(\left(I_{\mathbf{X}}-Q^{\circ}\right) \mathbf{T} B v,\left(I_{\mathbf{X}}-Q^{\circ}\right) \mathbf{T} B v\right) \\
& =s(\mathbf{T} B v, \mathbf{T} B v) \\
& =a(B v, B v)
\end{aligned}
$$

gives the square of the distance between $\mathbf{T} B v \in \mathbf{X}$ and the closed subspace $\mathbf{X}^{\circ}$. We define $T_{L}^{\rho}: \mathbf{H}_{a} \longrightarrow \mathbb{R}$ by

$$
T_{L}^{\rho}(v)=\frac{a(v, v)-\rho b(v, v)}{a(v, v)-2 \rho b(v, v)+\rho^{2} \min \left\{s(w, w): w \in \mathbf{T} B v+\mathbf{X}^{\circ}\right\}}
$$

for all $v \in \mathbf{H}_{\mathbf{a}}$. If the eigenvalues $\lambda_{ \pm i}^{\rho}$ are positive and represented in the form

$$
\lambda_{ \pm i}^{\rho}=\rho+\frac{\rho}{\tau_{ \pm i}^{\rho}-1} \quad \text { with } \tau_{ \pm i}^{\rho}=\frac{\lambda_{ \pm i}^{\rho}}{\lambda_{ \pm i}^{\rho}-\rho} \quad\left(i=1, \ldots, k_{\rho}^{ \pm}\right)
$$

then the quantities $\tau_{ \pm i}^{\rho} \in \mathbb{R}$ are characterized (see [13: Theorem 1.4 and Corollary 2.1]) by variational principles:

We have for $i \in\left\{1, \ldots, k_{\rho}^{-}\right\}$

$$
\begin{aligned}
& 0>\tau_{-i}^{\rho}=\inf _{\substack{V \subset \mathbf{H}_{\boldsymbol{a}} \\
\operatorname{dim} V=i \\
V \cap D_{p}=\{0\}}} \max _{0 \neq v \in V} T_{L}^{\rho}(v) \\
& =\inf _{\substack{V \subset \mathbf{H}_{a} \\
\operatorname{dim} V=i \\
V \cap D_{\rho}=\{0\}}} \max _{0 \neq v \in V} \min _{\substack{w \in \mathbf{X} \\
(w, T u)=b(v, u) \\
\forall u \in \mathbf{H}_{\mathbf{a}}}} \frac{a(v, v)-\rho b(v, v)}{a(v, v)-2 \rho b(v, v)+\rho^{2} s(w, w)}
\end{aligned}
$$

and for $i \in\left\{1, \ldots, k_{\rho}^{+}\right\}$

$$
\begin{aligned}
& 1<\tau_{+i}^{\rho}=\sup _{\substack{V \subset \mathbf{H}_{a} \\
\operatorname{dim} V=i \\
V \cap D_{\rho}=\{0\}}} \min _{0 \neq \nu \in V} T_{L}^{\rho}(v) \\
& =\sup _{\substack{V \subset \mathbf{H}_{\mathbf{a}} \\
\operatorname{dim} V=i \\
V \cap D_{\rho}=\{0\}}} \min _{0 \neq v \in V} \max _{\substack{w \in \mathbf{X} \\
0(\boldsymbol{w}, T u)=b(v, u) \\
\forall u \in \mathbf{H}_{\mathbf{a}}}} \frac{a(v, v)-\rho b(v, v)}{a(v, v)-2 \rho b(v, v)+\rho^{2} s(w, w)} .
\end{aligned}
$$


For a discretization of the eigenvalue problem (1.1), let $n \in \mathbb{N}$ and $m \in \mathbb{N}_{0}$ and suppose the following:

$(\mathrm{L})_{1} v_{1}, \ldots, v_{n} \in \mathbf{H}_{a}$ are linearly independent, $V_{n}=\operatorname{span}\left\{v_{1}, \ldots, v_{n}\right\}, S V_{n} \cap D_{\rho}=\{0\}$.

$(\mathbf{L})_{2} w_{1}^{\star}, \ldots, w_{n}^{\star} \in \mathbf{X}$ satisfy $s\left(w_{i}^{\star}, \mathbf{T} u\right)=b\left(v_{i}, u\right)$ for all $u \in \mathbf{H}_{a}(1 \leq i \leq n)$.

$(\mathrm{L})_{3} w_{0}^{\circ}, \ldots, w_{m}^{\circ} \in \mathbf{X}^{\circ}$ where $w_{0}^{\circ}=0$ and $w_{1}^{\circ}, \ldots, w_{m}^{\circ}$ are linearly independent.

Then, we construct matrices $A_{1}=\left(a_{i k}^{(1)}\right), A_{2}=\left(a_{i k}^{(2)}\right), A_{3}=\left(a_{i k}^{(3)}\right)$ by

$$
\begin{array}{ll}
a_{i k}^{(1)} & =a\left(v_{k}, v_{i}\right) \\
a_{i k}^{(2)} & =b\left(v_{k}, v_{i}\right) . \\
a_{i k}^{(3)} & =a\left(B v_{k}, B v_{i}\right)
\end{array}
$$

and matrices $C_{11}=\left(c_{i k}^{(11)}\right), C_{12}=\left(c_{i k}^{(12)}\right), C_{22}=\left(c_{i k}^{(22)}\right)$ by

$$
\begin{array}{ll}
c_{i k}^{(11)}=s\left(w_{k}^{\star}, w_{i}^{\star}\right) & (1 \leq i, k \leq n) \\
c_{i k}^{(12)}= \begin{cases}s\left(w_{k}^{\circ}, w_{i}^{*}\right) & \text { for } m>0 \\
0 & \text { for } m=0\end{cases} & (1 \leq i \leq n ; 1 \leq k \leq \max \{1, m\}) \\
c_{i k}^{(22)}= \begin{cases}s\left(w_{k}^{\circ}, w_{i}^{\circ}\right) & \text { for } m>0 \\
1 & \text { for } m=0\end{cases} & (1 \leq i, k \leq \max \{1, m\})
\end{array}
$$

and consider the matrix eigenvalue problem

$$
\left(A_{1}-\rho A_{2}\right) x=\tau\left(A_{1}-2 \rho A_{2}+\rho^{2}\left(C_{11}+C_{12} C_{22}^{-1} C_{12}^{H}\right)\right) x
$$

where $\tau \in \mathbb{R}$ and $x \in \mathbb{C}^{n}$. Denote all eigenvalues $\tau$ of problem (1.6) in $\mathbb{R} \backslash[0,1]$ by $\tau_{ \pm i}^{\rho[n, m]}$ and arrange them in the following order:

$$
\tau_{-1}^{\rho[n, m]} \leq \ldots \leq \tau_{-n^{-}}^{\rho[n, m]}<0<1<\tau_{+n^{+}}^{\rho[n, m]} \leq \cdots \leq \tau_{+1}^{\rho[n, m]}
$$

with $n^{-}, n^{+} \in \mathbb{N}_{0}$ and $n^{-}+n^{+} \leq n$. Then we obtain (see [13: Theorem 2.4]) the eigenvalue bounds

$$
\Lambda_{-i}^{\rho[n, m]}=\rho+\rho\left(\tau_{-i}^{\rho[n, m]}-1\right)^{-1} \leq \lambda_{-i}^{\rho} \quad\left(i=1, \ldots, \min \left\{n^{-}, k_{\rho}^{-}\right\}\right)
$$

and

$$
\Lambda_{+i}^{\rho[n, m]}=\rho+\rho\left(\tau_{+i}^{\rho[n, m]}-1\right)^{-1} \geq \lambda_{+i}^{\rho} \quad\left(i=1, \ldots, \min \left\{n^{+}, k_{\rho}^{+}\right\}\right) .
$$

We may assume that the eigenvectors of the matrix eigenvalue problem (1.6) constitute an orthonormal system with respect to the inner product

$$
\mathbb{C}^{n} \times \mathbb{C}^{n} \ni(x, y) \longmapsto x^{H}\left(A_{1}-2 \rho A_{2}+\rho^{2}\left(C_{11}-C_{12} C_{22}^{-1} C_{12}^{H}\right)\right) y \in \mathbb{C} .
$$

Now, if $\left(\tau_{ \pm i}^{\rho[n, m]}, x\right) \in \mathbb{R} \times \mathbb{C}^{n}$ with $x=\left(x_{1}, \ldots, x_{n}\right)^{t} \in \mathbb{C}^{n}$ is an eigenpair of problem (1.6), we set

$$
u_{ \pm i}^{\rho[n, m]}=\sum_{j=1}^{n} x_{j} v_{j} \in V_{n} \quad\left(i=1, \ldots, n^{ \pm}\right) .
$$

This orthonormalization is equivalent to

$$
a\left((I-\rho B) u_{k}^{\rho[n, m]}, u_{l}^{\rho[n, m]}\right)=\delta_{k l} \tau_{k}^{\rho[n, m]} .
$$

for $\left.k, l \in\left\{-1, \ldots,-n^{-},+n^{+}, \ldots,+1\right\}\right)$. 
Remark 1.1. The choice $m=0$ yields the procedure of Lehmann-Maehly (cf. [13: Remark 2.2]) given by $\mathbf{X}=\mathbf{H}_{a}, s(\cdot, \cdot)=a(\cdot, \cdot), \mathbf{T}=I$ and $\mathbf{X}^{\circ}=\{0\}$.

In our investigation of the convergence we assume for the sake of simplicity that $\rho \notin \sigma(A)$ and, consequently, $D_{\rho}=\{0\}$ (cf. Remark 3.5 for a more general choice of $\rho$ ). We set $\mathbf{X}_{m}^{\circ}=\operatorname{span}\left\{w_{1}^{\circ}, \ldots, w_{m}^{\circ}\right\}$. Suppose the additional assumptions

$$
\bigcup_{n \in \mathbf{N}} V_{n} \text { is dense in }\left(\mathbf{H}_{a},\|\cdot\|_{a}\right)
$$

and

$$
\bigcup_{m \in \mathbb{N}} \mathbf{X}_{m}^{\circ} \text { is dense in }\left(\mathbf{X}^{\circ},\|\cdot\|_{s}\right)
$$

to be fulfilled.

On the set $\mathbb{N} \times \mathbb{N}$ let an order $\prec$ be given by

$$
(\tilde{n}, \tilde{m}) \prec(n, m) \quad \text { if } \quad \tilde{n} \leq n \text { and } \tilde{m} \leq m \text {. }
$$

We denote the limits of nets $\mathbb{N} \times \mathbb{N} \longrightarrow \mathbb{R}$ and $\mathbb{N} \times \mathbb{N} \longrightarrow \mathbf{H}_{a}$ defined by this order by the symbol $\lim _{n, m \rightarrow \infty}$. Monotonicity is always related to this order. If $\left(n^{\prime}\right)$ and $\left(m^{\prime}\right)$ are subsequences of natural numbers, we denote the limits of the corresponding subnets by $\lim _{n^{\prime}, m^{\prime} \rightarrow \infty}$.

Theorem 1.2. Let $0<\rho \notin \sigma(A)$ and $\lambda_{-k_{p}^{-}}^{\rho}>0$. Then:

For $i \in\left\{1, \ldots, k_{\rho}^{-}\right\}$we have

$$
\lim _{n \rightarrow \infty} \Lambda_{-i}^{p[n, 0]}=\lambda_{-i}^{p}
$$

and

$$
\lim _{n, m \rightarrow \infty} \Lambda_{-i}^{\rho[n, m]}=\lambda_{-i}^{\rho}
$$

monotone increasing on $\mathbb{N}$ and $\mathbb{N} \times \mathbb{N}$, respectively.

For $i \in\left\{1, \ldots, k_{\rho}^{+}\right\}$we have

$$
\lim _{n \rightarrow \infty} \Lambda_{+i}^{\rho[n, 0]}=\lambda_{+i}^{\rho}
$$

and

$$
\lim _{n, m \rightarrow \infty} \Lambda_{+i}^{p[n, m]}=\lambda_{+i}^{\rho}
$$

monotone decreasing on $\mathbb{N}$ and $\mathbb{N} \times \mathbb{N}$, respectively.

The discretization (1.6) allows for the approximation of eigenelements corresponding to the eigenvalue $\lambda_{ \pm 1}^{\boldsymbol{p}}$. For that purpose we have to take into account the multiplicity of the eigenvalue $\lambda_{ \pm 1}^{\rho}$. We set

$$
J_{ \pm 1}^{\rho}=\left\{j \in \mathbb{N}: \lambda_{ \pm j}^{\rho}=\lambda_{ \pm 1}^{\rho}\right\}
$$


(If the eigenvalue $\lambda_{-1}^{\rho}$ and $\lambda_{+1}^{\rho}$ does not exist, the set of indices $J_{-1}^{\rho}$ and $J_{+1}^{\rho}$ is empty, respectively.) Then

$$
N_{ \pm 1}^{\rho}=\operatorname{span}\left\{u_{ \pm j}^{\rho}: j \in J_{ \pm 1}^{\rho}\right\} \subset \mathbf{H}_{a}
$$

is the corresponding eigenspace of the eigenvalue $\lambda_{ \pm 1}^{\rho}$. Its multiplicity is $\left|J_{ \pm 1}\right|=$ $\operatorname{dim} N_{ \pm 1}^{\rho}$. Denote by

$$
P_{ \pm 1}^{\rho}: \mathbf{H}_{a} \longrightarrow N_{ \pm 1}^{\rho}
$$

the $a(\cdot, \cdot)$-orthogonal projection of $\mathbf{H}_{a}$ onto $N_{ \pm 1}^{\rho}$.

The following constants result from the position of $\rho \notin \sigma(A)$ relative to the spectrum $\sigma(A)$ of (1.1). Assume that $\sigma(A) \backslash\left\{\lambda_{ \pm 1}^{\rho}\right\} \neq \emptyset$ and set

$$
\begin{aligned}
& \delta^{\rho}=\min \{|\rho-\lambda|: \lambda \in \sigma(A)\} \\
& \gamma_{-}^{\rho}=\min \left\{\frac{\lambda}{\lambda-\rho}-\frac{\lambda_{-1}^{\rho}}{\lambda_{-1}^{\rho}-\rho}: \lambda \in \sigma(A)\right\} \quad \text { if } J_{-1}^{\rho} \neq \emptyset \\
& \gamma_{+}^{\rho}=\min \left\{\frac{\lambda_{+1}^{\rho}}{\lambda_{+1}^{\rho}-\rho}-\frac{\lambda}{\lambda-\rho}: \lambda \in \sigma(A)\right\} \quad \text { if } J_{+1}^{\rho} \neq \emptyset .
\end{aligned}
$$

Theorem 1.3. Let $0<\rho \notin \sigma(A)$ and $\lambda_{-1}^{\rho}>0$. Then:

For $i, j \in J_{ \pm 1}^{\rho}$ we have the limits

$$
\left.\begin{array}{r}
\lim _{n \rightarrow \infty}\left\|\left(I-P_{ \pm 1}^{\rho}\right) u_{ \pm i}^{\rho[n, 0]}\right\|_{a}=0 \\
\lim _{n \rightarrow \infty} a\left(\left(\tau_{ \pm i}^{\rho[n, 0]}\right)^{-1} u_{ \pm i}^{\rho[n, 0]},\left(\tau_{ \pm j}^{\rho[n, 0]}\right)^{-1} u_{ \pm j}^{\rho[n, 0]}\right)=\delta_{i j}
\end{array}\right\}
$$

and

$$
\left.\begin{array}{c}
\lim _{n, m \rightarrow \infty}\left\|\left(I-P_{ \pm 1}^{\rho}\right) u_{ \pm i}^{\rho[n, m]}\right\|_{a}=0 \\
\lim _{n, m \rightarrow \infty} a\left(\left(\tau_{ \pm i}^{\rho[n, m]}\right)^{-1} u_{ \pm i}^{\rho[n, m]},\left(\tau_{ \pm j}^{\rho[n, m]}\right)^{-1} u_{ \pm j}^{\rho[n, m]}\right)=\delta_{i j}
\end{array}\right\}
$$

For $m \in \mathbb{N}_{0}$ and sufficiently large $n \in \mathbb{N}$ we have the quantitative estimates

$$
\left\|\left(I-P_{-1}^{\rho}\right) u_{-j}^{\rho[n, m]}\right\|_{a}^{2} \leq\left(1+\frac{\rho}{\delta \rho}\right)^{2} \frac{1}{\gamma_{-}^{\rho}}\left[\tau_{-j}^{\rho[n, m]}-\tau_{-1}^{\rho}\right] \quad\left(j \in J_{-1}^{\rho}\right)
$$

and

$$
\left\|\left(I-P_{+1}^{\rho}\right) u_{+j}^{\rho[n, m]}\right\|_{a}^{2} \leq\left(1+\frac{\rho}{\delta^{\rho}}\right)^{2} \frac{1}{\gamma_{+}^{\rho}}\left[\tau_{+1}^{\rho}-\tau_{+j}^{\rho[n, m]}\right] \quad\left(j \in J_{+1}^{\rho}\right) .
$$

Remark 1.4. We can not expect the convergence of the sequence $\left(u_{ \pm i}^{\rho[n, 0]}\right)$ and of the net $\left(u_{ \pm i}^{\rho[n, m]}\right)$. The approximations $u_{ \pm i}^{\rho[n, m]}$ are not determined uniquely, since they depend (1.8) on eigenvectors of a matrix eigenvalue problem.

Instead, we find 
Corollary 1.5. There exists a subsequence $\left(n^{\prime}\right)$ in $\mathbb{N}$, a subnet $\left(n^{\prime} ; m^{\prime}\right)$ in $\mathbb{N} \times \mathbb{N}$ and an $a(\cdot, \cdot)$-orthonormal basis

$$
\left\{\Phi_{ \pm j}^{\rho} \in N_{ \pm 1}^{\rho}: j \in J_{ \pm 1}^{\rho}\right\}
$$

of $N_{ \pm 1}^{\rho}$ such that we have for $j \in J_{ \pm 1}^{\rho}$

$$
\lim _{n^{\prime} \rightarrow \infty}\left(\tau_{ \pm j}^{\rho\left[n^{\prime}, 0\right]}\right)^{-1} u_{ \pm j}^{\rho\left[n^{\prime}, 0\right]}=\Phi_{ \pm j}
$$

and

$$
\lim _{n^{\prime}, m^{\prime} \rightarrow \infty}\left(\tau_{ \pm j}^{\rho\left[n^{\prime}, m^{\prime}\right]}\right)^{-1} u_{ \pm j}^{\rho\left[n^{\prime}, m^{\prime}\right]}=\Phi_{ \pm j}
$$

In the following sections we shall prove Theorems 1.2 and 1.3 and Corollary 1.5. We restrict our considerations to the eigenvalues $\lambda_{-i}^{\rho}\left(1 \leq i \leq k_{\rho}^{-}\right.$with $\left.k_{\rho}^{-} \geq 1\right)$ which we assume to exist.

\section{Preliminary results}

Due to our assumptions, we have $0, \rho \notin \sigma(A)$. In particular,

$$
T=A(A-\rho I)^{-1} \in \mathcal{L}\left(\mathbf{H}_{a}\right)
$$

is a bounded and self-adjoint operator. Furthermore $T$ is bijective with $T^{-1}=I-$ $\rho B \in \mathcal{L}\left(\mathbf{H}_{a}\right)$. Evidently, $\left(\lambda_{-i}^{\rho}, u_{-i}^{\rho}\right) \in \mathbb{R} \times \mathbf{H}_{a}$ is an eigenpair of (1.1) if and only if $\left(\tau_{-i}^{\rho}, u_{-i}^{\rho}\right) \in \mathbb{R} \times \mathbf{H}_{a}$ is an eigenpair of $T \in \mathcal{L}\left(\mathbf{H}_{a}\right)$ with $\tau_{-i}^{\rho}=\frac{\lambda_{-i}^{\rho}}{\lambda_{-i}^{\rho}-\rho}$ for $i \in\left\{1, \ldots, k_{\rho}^{-}\right\}$. The spectrum of $T$ is bounded from below and begins with isolated eigenvalues

$$
\min \sigma(T)=\tau_{-1}^{\rho} \leq \tau_{-2}^{\rho} \leq \cdots \leq \tau_{-k_{\rho}^{-}}^{\rho}<0
$$

of finite multiplicity. These eigenvalues are characterized by the Poincaré principle

$$
\tau_{-i}^{\rho}=\min _{\substack{W \mathcal{W}_{a} \\ \text { dimw } w i}} \max _{0 \neq w \in W} \frac{a(T w, w)}{a(w, w)} \quad\left(i=1, \ldots, k_{\rho}^{-}\right)
$$

where the minimum is attained for $W=\operatorname{span}\left\{u_{-1}^{\rho}, \ldots, u_{-i}^{\rho}\right\}$. We set

$$
W_{n}=(I-\rho B)\left(V_{n}\right) \subset \mathbf{H}_{a} \quad(n \in \mathbb{N}) .
$$

According to (1.10),

$$
\bigcup_{n \in \mathbf{N}} W_{n} \text { is dense in }\left(\mathbf{H}_{a},\|\cdot\|_{a}\right)
$$

too. We denote by

$$
Q_{n}: \mathbf{H}_{a} \longrightarrow W_{n}
$$

the projection which maps $\mathbf{H}_{a}$ orthogonal with respect to the inner product $a(\cdot, \cdot)$ onto $W_{n}$, and by

$$
Q_{m}^{\circ}: \mathbf{X} \longrightarrow \mathbf{X}_{m}^{\circ}
$$

the projection, which maps $\mathbf{X}$ orthogonal with respect to the inner product $s(\cdot, \cdot)$ onto $\mathbf{X}_{m}^{\circ}$.

The assumptions (2.3) and (1.11) provide (cf. [8: Satz 1.1]) immediately 
Lemma 2.1. For all relatively compact subsets $M \subset \mathbf{H}_{a}$ we have

$$
\sup _{v \in M}\left\|\left(I-Q_{n}\right) v\right\|_{a} \rightarrow 0 \quad \text { as } n \rightarrow \infty
$$

$B y u_{n} \in \mathbf{H}_{a}\left(n \in \mathbb{N}_{0}\right)$ with $\left\|u_{n}-u_{0}\right\|_{a} \rightarrow 0$ as $n \rightarrow \infty$, it follows that

$$
\left\|Q_{n} u_{n}-u_{0}\right\|_{a} \rightarrow 0 \quad \text { as } n \rightarrow \infty
$$

For $w \in \mathbf{X}$ we have

$$
\left\|Q_{m}^{\circ} w-Q^{\circ} w\right\|_{s}=\inf _{w^{\prime} \in \mathbf{X}_{m}^{\circ}}\left\|w^{\prime}-Q^{\circ} w\right\|_{s} \rightarrow 0 \quad \text { as } m \rightarrow \infty
$$

Lemma 2.2. For $i \in \mathbb{N}$ let $U^{(i)} \subset \mathbf{H}_{a}$ be a subspace with $\operatorname{dim} U^{(i)}=\ddot{i}$. Then for all sufficiently large $n \in \mathbb{N}$ we have also $\operatorname{dim} Q_{n}\left(U^{(i)}\right)=i$.

Proof. For $u \in \mathbf{H}_{a}$ the identity

$$
\left\|\left(I-Q_{n}\right) u\right\|_{a}^{2}=\|u\|_{a}^{2}-\left\|Q_{n} u\right\|_{a}^{2}
$$

holds. Based on the compactness of the set $M=\left\{u \in U^{(i)}:\|u\|_{a}=1\right\}$ in $\left(\mathbf{H}_{a},\|\cdot\|_{a}\right)$, it follows from (2.4) that

$$
\lim _{n \rightarrow \infty} \sup _{u \in M}\left(1-\left\|Q_{n} u\right\|_{a}^{2}\right)=0
$$

Hence, for sufficiently large $n \in \mathbb{N}$ the projection $Q_{n}: U^{(i)} \rightarrow \mathbf{H}_{a}$ is injective. Thus, the assertion holds

Based on the assumptions $(L)_{1}-(L)_{3}$ of the discretization, we define for $(n, m) \in$ $\mathbb{N} \times \mathbb{N}_{0}$ the Goerisch mapping $G_{m}: V_{n} \rightarrow \mathrm{X}$ as follows:

If $v=\sum_{j=1}^{n} x_{j} v_{j} \in V_{n}$ with $x=\left(x_{1}, \ldots, x_{n}\right)^{t} \in \mathbb{C}^{n}$ is given, let be $w^{\star}=$ $\sum_{j=1}^{n} x_{j} w_{j}^{\star} \in \mathrm{X}$ and

$$
G_{m}(v)= \begin{cases}\mathbf{T} B v & \text { for } m=0 \\ w^{\star}-Q_{m}^{\circ} w^{\star} & \text { for } m \geq 1\end{cases}
$$

Then, we have (cf. [13: Lemma 2.3]) for such $v \in V_{n}$

$$
\begin{aligned}
x^{H}\left(C_{11}-C_{12} C_{22}^{-1} C_{12}^{H}\right) x & =\min \left\{s\left(w^{\star}+w, w^{\star}+w\right): w \in \mathbf{X}_{m}^{\circ}\right\} \\
& =s\left(G_{m} v, G_{m} v\right) \\
& \geq x^{H} A_{3} x=a(B v, B v)=s(\mathbf{T} B v, \mathbf{T} B v)
\end{aligned}
$$

Consequently, for $n \in \mathbb{N}$ and $v \in V_{n}$ the sequence $\left(\left\|G_{m} v\right\|_{s}\right)_{m \in \mathbb{N}}$ is monotone decreasing.

For the $(n \times n)$-matrix $A_{3, m}=C_{11}-C_{12} C_{22}^{-1} C_{12}^{H}$ the following limit (cf. [4: Theorem 4] and [12: Section 7]) can be established. 
Lemma 2.3. Suppose $n \in \mathbb{N}, x=\left(x_{1}, \ldots, x_{n}\right)^{t} \in \mathbb{C}^{n}$ and $v=\sum_{j=1}^{n} x_{j} v_{j} \in V_{n}$. Then we have

$$
x^{H}\left(A_{3, m}-A_{3}\right) x=\left\|G_{m} v-\mathbf{T} B v\right\|_{3}^{2} \downarrow 0 \quad \text { as } m \rightarrow \infty .
$$

The convergence $\lim _{m \rightarrow \infty} G_{m} v=\mathbf{T} B v$ is uniformly on all bounded subsets of $V_{n}$.

Proof. Because of $G_{m} v-\mathbf{T} B v, w^{\star}-\mathbf{T} B v \in \mathbf{X}^{\circ}$, the limit (2.6) yields

$$
\begin{aligned}
0 & \leq s\left(G_{m} v, G_{m} v\right)-s(\mathbf{T} B v, \mathbf{T} B v) \\
& =s\left(G_{m} v-\mathbf{T} B v, G_{m} v-\mathbf{T} B v\right) \\
& =s\left(G_{m} v, G_{m} v-\mathbf{T} B v\right) \\
& \downarrow s\left(\left(I_{\mathbf{X}}-Q^{\circ}\right) w^{\star}, Q^{\circ}\left(w^{\star}-\mathbf{T} B v-w^{\star}\right)\right) \\
& =0
\end{aligned}
$$

as $m \rightarrow \infty$

For $n \in \mathbb{N}$ and $i \in\left\{1, \ldots, n^{-}\right\}$the elements in (1.8) are normalized by

$$
\left\|(I-\rho B) u_{-i}^{\rho[n, 0]}\right\|_{a}^{2}=1
$$

and

$$
\left\|(I-\rho B) u_{-i}^{\rho[n, m]}\right\|_{a}^{2}+\rho^{2}\left\|\left(G_{m}-\mathrm{T} B\right) u_{-i}^{\rho[n, m]}\right\|_{s}^{2}=1 \quad(m \in \mathbb{N}) .
$$

By the boundedness of the sequence $\left(u_{-i}^{p[n, m]}\right)_{m \in N}$ in $V_{n}$ Lemma 2.3 yields immediately

Lemma 2.4. For $i \in\left\{1, \ldots, n^{-}\right\}$we have

$$
\lim _{m \rightarrow \infty}\left\|(I-\rho B) u_{-i}^{\rho[n, m]}\right\|_{a}=1 \text {. }
$$

For $n \in \mathbb{N}, x=\left(x_{1}, \ldots, x_{n}\right)^{t} \in \mathbb{C}^{n}$ and $v=\sum_{j=1}^{n} x ; v_{j} \in V_{n}$ the Rayleigh quotient of the matrix eigenvalue problem (1.6) is given by

$$
T_{L, m}^{\rho}(v)=\frac{a((I-\rho B) v, v)}{\|(I-\rho B) v\|_{a}^{2}+\rho^{2}\left\|\left(G_{m}-\mathrm{T} B\right) v\right\|_{s}^{2}} \quad\left(m \in \mathbb{N}_{0}\right) .
$$

Hence, for $v \in V_{n}$ with $a((I-\rho B) v, v)<0$ the monotonicity in (2.8) provides

$$
0>T_{L, m}^{\rho}(v) \geq T_{L, m+1}^{\rho}(v) \geq T_{L, 0}^{\rho}(v) \quad(m \in \mathbb{N}) .
$$

Since for $v \in V_{n}$ the sign of $T_{L, m}^{\rho}(v)$ does not depend on $m \in \mathbb{N}_{0}$, we have

Lemma 2.5. The number of negative eigenvalues of the matrix eigenvalue problem (1.6) is for $m=0$ equal to that for $m \geq 1$.

We have to introduce an additional notation of the eigenvalues of the matrix eigenvalue problem (1.6). Let

$$
\tilde{\tau}_{1}^{\rho[n, m]} \leq \tilde{\tau}_{2}^{\rho[n, m]} \leq \cdots \leq \tilde{\tau}_{n}^{\rho[n, m]} \quad\left((n, m) \in \mathbb{N} \times \mathbb{N}_{0}\right)
$$


be all eigenvalues of (1.6) arranged in monotone increasing order. According to (1.8), we define the elements $\tilde{u}_{i}^{\rho[n, m]} \in V_{n}(i=1, \ldots, n)$. If $\tilde{\tau}_{i}^{\rho[n, m]}<0$ holds, then with respect to (1.7) and (1.8) we have $\left(\tilde{\tau}_{i}^{\rho[n, m]}, \tilde{u}_{i}^{\rho[n, m]}\right)=\left(\tau_{-i}^{\rho[n, m]}, u_{-i}^{\rho[n, m]}\right) \in \mathbb{R} \times V_{n}$.

Poincaré's principle applied to the eigenvalues in (2.13) now gives the following characterization:

$$
\tilde{\tau}_{i}^{\rho[n, m]}=\min _{\substack{V \subset V_{n} \\ d i m V=i}} \max _{0 \neq v \in V} T_{L, m}^{\rho}(v)=T_{L, m}^{\rho}\left(\tilde{u}_{i}^{\rho[n, m]}\right) \quad(i=1, \ldots, n) .
$$

In the special case $m=0$ (Lehmann-Maehly method) we substitute $v=T w$ and obtain from (2.14)

$$
\tilde{\tau}_{i}^{p[n, 0]}=\min _{\substack{W \subset W_{n} \\ d i m w=i}} \max _{0 \neq w \in W} \frac{a(T w, w)}{a(w, w)} \quad(i=1, \ldots, n) .
$$

Consequently, we have (cf. [10]) proved

Theorem 2.6. For $i \in\left\{1, \ldots, k_{\rho}^{-}\right\}$and $n \in \mathbb{N}$ with $n \geq k_{\rho}^{-}$we have

$$
\tau_{-i}^{\rho} \leq \tilde{\tau}_{i}^{\rho[n, 0]} .
$$

Moreover, the quantities $\tilde{\tau}_{i}^{p[n, 0]} \in \mathbb{R}$ are upper Rayleigh-Ritz bounds to the eigenvalues $\tau_{-i}^{\rho}$ of the operator $T \in \mathcal{L}\left(\mathbf{H}_{a}\right)$ with respect to the trial functions $(I-\rho B) v_{1}, \ldots,(I-$ $\rho B) v_{n} \in \mathbf{H}_{a}$.

\section{The convergence of the eigenvalue approximations}

In order to prove Theorem 1.2, we first give some results on the eigenvalues of the matrix eigenvalue problem (1.6).

Lemma 3.1. For $m \in \mathbb{N}_{0}$ and $i \in\{1, \ldots, n\}$ we have

$$
\tilde{\tau}_{i}^{\rho[n+1, m]} \leq \tilde{\tau}_{i}^{\rho[n, m]} \quad(n \in \mathbb{N})
$$

Proof. Again, the Poincaré principle (2.14) provides

$$
\tilde{r}_{i}^{\rho[n+1, m]}=\min _{\substack{v \subset V_{n+1} \\ \operatorname{dim} V=i}} \max _{\substack{0 \neq v \in V \\ x_{L, m}}} T_{L}^{\rho}(v)
$$

For the particular subspace

$$
U^{(i)}=\operatorname{span}\left\{\tilde{u}_{1}^{\rho[n, m]}, \ldots, \tilde{u}_{i}^{\rho[n, m]}\right\} \subset V_{n} \subset V_{n+1}
$$

we have

$$
\tilde{\tau}_{i}^{\rho[n+1, m]} \leq \max _{0 \neq v \in U^{(i)}} T_{L, m}^{\rho}(v)=\tilde{\tau}_{i}^{\rho[n, m]}
$$

and the assertion is proved 
Lemma 3.2 For $i \in\left\{1, \ldots, k_{\rho}^{-}\right\}$we have monotone decreasing

$$
\lim _{n \rightarrow \infty} \tilde{\tau}_{i}^{\rho[n, 0]}=\tau_{-i}^{\rho}<0 .
$$

Proof (cf. [8: Satz 2.2]). We set $U^{(i)}=\operatorname{span}\left\{u_{-1}^{\rho}, \ldots, u_{-i}^{\rho}\right\} \subset \mathbf{H}_{a}$. For all sufficiently large $n \in \mathbb{N}$ Lemma 2.2 gives $\operatorname{dim} Q_{n}\left(U^{(i)}\right)=i$. For those $n \in \mathbb{N}$ we obtain by (2.15) and (2.16)

$$
\tau_{-i}^{\rho} \leq \tilde{\tau}_{i}^{\rho[n, 0]} \leq \max _{\substack{u \in U^{(i)} \\\|u\|_{a}=1}} \frac{a\left(T Q_{n} u, Q_{n} u\right)}{a\left(Q_{n} u, Q_{n} u\right)}=\frac{a\left(T Q_{n} u_{n}, Q_{n} u_{n}\right)}{a\left(Q_{n} u_{n}, Q_{n} u_{n}\right)}
$$

At this, we assume the maximum to be attained by the point $u_{n} \in\left\{u \in U^{(i)}:\|u\|_{a}=\right.$ 1). According to $(3.1)$ the sequence $\left(\tilde{\tau}_{i}^{\rho \mid n, 0]}\right)_{n \in N}$ is monotone decreasing. Now, we shall prove the existence of a subsequence converging to $\tau_{-i}^{\rho}$. By the equivalence of all norms on $U^{(i)}$ and by the boundedness of the sequence $\left(u_{n}\right)$, there exists a subsequence $\left(u_{n^{\prime}}\right)$ with

$$
\lim _{n^{\prime} \rightarrow \infty}\left\|u_{n^{\prime}}-u_{0}\right\|_{a}=0 \quad \text { for any } u_{0} \in U^{(i)} \text { with }\left\|u_{0}\right\|_{a}=1
$$

Due to (2.5), we have $\lim _{n^{\prime} \rightarrow \infty}\left\|Q_{n^{\prime}} u_{n^{\prime}}-u_{0}\right\|_{a}=0$. Consequently, (2.2) gives

$$
\tau_{-i}^{\rho} \leq \lim _{n^{\prime} \rightarrow \infty} \tilde{\tau}_{-i}^{\rho\left(n^{\prime}, 0\right)} \leq \frac{a\left(T u_{0}, u_{0}\right)}{a\left(u_{0}, u_{0}\right)} \leq \tau_{-i}^{\rho}
$$

and the assertion is proved

Because of the monotone convergence (3.2), we always have $\tilde{\tau}_{k_{p}^{-}}^{\rho[n, 0]}<0$ for all sufficiently large $n \in \mathbb{N}$. Due to Lemma 2.5, for those $n \in \mathbb{N}$ and $i \in\left\{1, \ldots, k_{\rho}^{-}\right\}$the notation (2.13) of the eigenvalues of (1.6) is now superfluous. Then, $n^{-} \geq k_{\rho}^{-}$holds in the arrangement (1.7).

Lemma 3.3 For all sufficiently large $n \in \mathbb{N}$ and $i \in\left\{1, \ldots, k_{p_{-}}^{-}\right\}$we have

$$
\tau_{-i}^{\rho} \leq \tau_{-i}^{\rho[n, 0]} \leq \tau_{-i}^{\rho[n, m+1]} \leq \tau_{-i}^{\rho[n, m]}<0 \quad(m \in \mathbb{N})
$$

and

$$
\lim _{m \rightarrow \infty} \tau_{-i}^{\rho[n, m]}=\tau_{-i}^{\rho[n, 0]}
$$

Proof. Lemma 2.5, Poincaré's principle (2.14) and the monotonicity in (2.12) give the estimates

$$
\begin{aligned}
& 0>\tau_{-i}^{\rho[n, m]}=\min _{\substack{v \subset V_{n} \\
d i m v i}} \max _{0 \neq v \in V} T_{L, m}^{\rho}(v) \\
& \geq \min _{\substack{v \mathcal{L} V_{n} \\
d i m v=i}} \max _{0 \neq v \in V} T_{L, m+1}^{\rho}(v)=\tau_{-i}^{\rho[n, m+1]}
\end{aligned}
$$

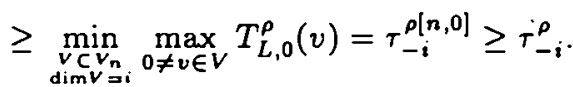

Since eigenvalues depend continuously on the elements of its matrix, (3.4) follows by (2.8) 
Now, (3.2) and (3.4) give

Theorem 3.4. For $i \in\left\{1, \ldots, k_{\rho}^{-}\right\}$we have

$$
\lim _{n \rightarrow \infty} \tau_{-i}^{\rho[n, 0]}=\tau_{-i}^{\rho}
$$

and

$$
\lim _{n, m \rightarrow \infty} \tau_{-i}^{\rho[n, m]}=\tau_{-i}^{\rho}
$$

monotone decreasing on $\mathbb{N}$ and $\mathbb{N} \times \mathbb{N}$, respectively.

Proof of Theorem 1.2. By the transformation $\tau \longmapsto \rho+\frac{\rho}{\tau-1}$ Theorem 3.4 provides the assertions

Remark 3.5. The assertions of Theorem 1.2 are also valid if $\rho \in \sigma(A)$ is an isolated eigenvalue of (1.1) of arbitrary multiplicity.

In this case,

$$
D_{\rho}=\{v \in \mathcal{D}(A):(A-\rho I) v=0\} \neq\{0\}
$$

is a non-trivial closed subspace. We denote by $\mathbf{H}_{a}^{\rho}=\mathbf{H}_{a} \ominus D_{\rho}$ the $a(\cdot, \cdot)$-orthogonal complement of $D_{\rho}$ in $\mathbf{H}_{a}$ and by $P^{\rho}: \mathbf{H}_{a} \longrightarrow \mathbf{H}_{a}^{\rho}$ the corresponding orthogonal projection. Instead of (1.10), suppose now

$$
\bigcup_{n \in \mathbb{N}} P^{\rho}\left(V_{n}\right) \text { is dense in }\left(\mathbf{H}_{a}^{\rho},\|\cdot\|_{a}\right) \text {. }
$$

Since $\mathbf{H}_{\mathbf{a}}^{\rho}$ forms a reducing subspace of $A$, the operator

$$
T=A\left(\left.(A-\rho I)\right|_{\mathbf{H}_{a}^{\rho}}\right)^{-1} \in \mathcal{L}\left(\mathbf{H}_{a}^{\rho}\right)
$$

is bounded and self-adjoint. $\left(\lambda_{-i}^{\rho}, u_{-i}^{\rho}\right) \in \mathbb{R} \times \mathbf{H}_{a}$ is an eigenpair of (1.1) if and only if $\left(\tau_{-i}^{\rho}, u_{-i}^{\rho}\right) \in \mathbb{R} \times \mathbf{H}_{a}^{\rho}$ is an eigenpair of $T \in \mathcal{L}\left(\mathbf{H}_{a}^{\rho}\right)$ for $i \in\left\{1, \ldots, k_{\rho}^{-}\right\}$. Obviously, we have for $v \in \mathcal{D}(A)$ the equation

$$
(I-\rho B) v=A^{-1}(A-\rho I) v=(I-\rho B) P^{\rho} v .
$$

Since $\mathcal{D}(A)$ is dense in $\mathbf{H}_{\mathbf{a}}$, we have for all $v \in \mathbf{H}_{\mathbf{a}}$

$$
a((I-\rho B) v, v)=a\left((I-\rho B) P^{\rho} v, P^{\rho} v\right)
$$

and

$$
\|(I-\rho B) v\|_{a}=\left\|(I-\rho B) P^{\rho} v\right\|_{a}
$$

and thus

$$
T_{L, 0}^{\rho}(v)=T_{L, 0}^{\rho}\left(P^{\rho} v\right)
$$

Due to assumption $(\mathrm{L})_{1}$, the projection $P^{\rho}: V_{n} \longrightarrow \mathbf{H}_{a}^{\rho}$ is injective. Setting

$$
W_{n}=(I-\rho B) P^{\rho}\left(V_{n}\right) \subset \mathbf{H}_{a}^{\rho} \quad(n \in \mathbb{N})
$$

Lemma 3.2 holds in this situation, too. 


\section{Convergence of the eigenelement approximations}

The eigenspace $N_{-1}^{\rho}$ corresponds to the eigenvalue $\tau_{-1}^{\rho}$ of the operator $T \in \mathcal{L}\left(\mathbf{H}_{a}\right)$. Since $N_{-1}^{\rho}$ is a reducing subspace of $A$, the operator $T$ and the projection $P_{-1}^{\rho}$ commute. The distance of the eigenvalue $\tau_{-1}^{\rho}$ to the next spectral point of $T$ is given by (1.15).

We denote by $\left\{E_{\lambda} \in \mathcal{L}\left(\mathbf{H}_{a}\right): \lambda \in \mathbb{R}\right\}$ the continuous (to the left) spectral family of $T$. Then the following estimation of the distance of an element $v \in \mathbf{H}_{a}$ to its best approximation in $N_{-1}^{\rho}$ holds (cf. the more general result [9: Satz 2.3]).

Lemma 4.1. For $v \in \mathbf{H}_{a}$ we have

$$
\left\|\left(I-P_{-1}^{\rho}\right) v\right\|_{a}^{2} \leq \frac{1}{\gamma_{-}^{\rho}}\left[a(T v, v)-\tau_{-1}^{\rho} a(v, v)\right] .
$$

Proof. Due to the spectral theorem, we have for $v \in \mathbf{H}_{\mathbf{a}}$

$$
\begin{aligned}
a(T v, v) & =\tau_{-1}^{\rho} a\left(P_{-1}^{\rho} v, v\right)+\int_{\tau_{-1}^{\rho}+\gamma_{-}^{\rho}}^{\|T\|+0} \lambda d a\left(E_{\lambda} v, v\right) \\
& \geq \tau_{-1}^{\rho} a\left(P_{-1}^{\rho} v, v\right)+\left(\tau_{-1}^{\rho}+\gamma_{-}^{\rho}\right) \int_{\tau_{-1}^{+}+\gamma_{-}^{\rho}}^{\|T\|+0} d a\left(E_{\lambda} v, v\right) \\
& =\tau_{-1}^{\rho} a\left(P_{-1}^{\rho} v, v\right)+\left(\tau_{-1}^{\rho}+\gamma_{-}^{\rho}\right)\left[a(v, v)-a\left(P_{-1}^{\rho} v, v\right)\right] \\
& =\tau_{-1}^{\rho} a(v, v)+\gamma_{-}^{\rho} a\left(\left(I-P_{-1}^{\rho}\right) v, v\right)
\end{aligned}
$$

and the assertion is proved

Setting $u, v \in \mathbf{H}_{a}$ with $v=(I-\rho B) u \neq 0$, the equation $\frac{a(T v, v)}{a(v, v)}=T_{L, 0}^{\rho}(u)$ holds and (4.1) provides

$$
\left\|\left(I-P_{-1}^{\rho}\right) v\right\|_{a}^{2} \leq \frac{a(v, v)}{\gamma_{-}^{\rho}}\left[T_{L, 0}^{\rho}(u)-\tau_{-1}^{\rho}\right] .
$$

Proof of Theorem 1.3. In particular, we now set for $m \in \mathbb{N}_{0}$ and sufficiently large $n \in \mathbb{N}$

$$
v_{-j}^{\rho[n, m]}=(I-\rho B) u_{-j}^{\rho[n, m]} \quad\left(j \in J_{-1}^{\rho}\right) .
$$

Then, according to $(2.9)_{a}$ and $(2.9)_{b}$ we find

$$
a\left(v_{-j}^{\rho[n, m]}, v_{-j}^{\rho[n, m]}\right) \leq 1 \quad\left(j \in J_{-1}^{\rho}\right)
$$

and

$$
0>\tau_{-j}^{\rho[n, m]}=T_{L, m}^{\rho}\left(u_{-j}^{\rho[n, m]}\right) \geq T_{L, 0}^{\rho}\left(u_{-j}^{\rho[n, m]}\right) .
$$

Consequently, (4.2) yields

$$
\left\|\left(I-P_{-1}^{\rho}\right) v_{-j}^{\rho[n, m]}\right\|_{a}^{2} \leq \frac{1}{\gamma_{-}^{\rho}}\left[\tau_{-j}^{\rho[n, m]}-\tau_{-1}^{\rho}\right] \quad\left(j \in J_{-1}^{\rho}\right) .
$$


Now, Theorem 3.4' gives :

$$
\lim _{n \rightarrow \infty}\left\|\left(I-P_{-1}^{\rho}\right) v_{-j}^{\rho[n, 0]}\right\|_{a}=0 \quad\left(j \in J_{-1}^{\rho}\right)
$$

and

$$
\lim _{n, m \rightarrow \infty}\left\|\left(I-P_{-1}^{\rho}\right) v_{-j}^{\rho[n, m]}\right\|_{a}=0 \quad\left(j \in J_{-1}^{\rho}\right) .
$$

By (4.3), we have $(A-\rho I)^{-1} v_{-j}^{\rho[n, m]}=B u_{-j}^{\rho[n, m]}$. The operators $(A-\rho I)^{-1}$ and $B$ and the projection $I-P_{-1}^{\rho}$ commute. Hence, $(4.4)_{b}$ provides for $j \in J_{-1}^{\rho}$.

$$
B\left(I-P_{-1}^{\rho}\right) u_{-j}^{\rho[n, m]}=(A-\rho I)_{,}^{-1}\left(I-P_{-1}^{\rho}\right) \hat{v}_{-j}^{\rho[n, m]} \longrightarrow 0
$$

as $n, m \rightarrow \infty$ and finally

$$
\left(I-P_{-1}^{\rho}\right) u_{-j}^{\rho[n, m]}=\left(I-P_{-1}^{\rho}\right) v_{-j}^{\rho[n, m]}+\rho \dot{B}\left(I-P_{-1}^{\rho}\right) u_{-j}^{\rho[n, m]} \longrightarrow 0
$$

as $n, m \rightarrow \infty$. The limits (2.10) and (4.4)b give

$$
\begin{aligned}
\left(1-\frac{\rho}{\lambda_{-1}^{\rho}}\right)^{2}\left\|P_{-1}^{\rho} u_{-j}^{\rho[n, m]}\right\|_{a}^{2} & =\left\|(I-\rho B) P_{-1}^{\rho} u_{-j}^{\rho[n, m]}\right\|_{a}^{2} \\
& =\left\|P_{-1}^{\rho} v_{-j}^{\rho[n, m]}\right\|_{a}^{2} \\
& =\left\|v_{-j}^{\rho[n, m]}\right\|_{a}^{2}-\left\|\left(I-P_{-1}^{\rho}\right) v_{-j}^{\rho[n, m]}\right\|_{a}^{2} \\
& \rightarrow 1
\end{aligned}
$$

as $n, m \rightarrow \infty$. Consequently, we have

$$
\left\|P_{-1}^{\rho} u_{-j}^{\rho[n, m]}\right\|_{a}^{2} \longrightarrow\left(\frac{\lambda_{-1}^{\rho}}{\lambda_{-1}^{\rho}-\rho}\right)^{2}=\left(\tau_{-1}^{\rho}\right)^{2}
$$

as $n, m \rightarrow \infty$ and, by (4.6),

$$
\left\|u_{-j}^{\rho[n, m]}\right\|_{a}^{2} \longrightarrow\left(\tau_{-1}^{\rho}\right)^{2}
$$

as $n, m \rightarrow \infty$. With the aid of $(1.9),(2.10),(3.5)_{b}$ and (4.8) we arrive for $i, j \in J_{-1}^{\rho}$ with $i \neq j$ at

$$
\begin{aligned}
& \left|a\left(\frac{1}{\tau_{-1}^{\rho}} u_{-i}^{\rho[n, m]}, u_{-j}^{\rho[n, m]}\right)\right| \\
& \quad=\left|a\left(\frac{1}{\tau_{-1}^{\rho}} u_{-i}^{\rho[n, m]}-v_{-i}^{\rho[n, m]}, u_{-j}^{\rho[n, m]}\right)\right| \\
& \quad \leq\left\|u_{-j}^{\rho[n, m]}\right\|_{a}\left\|\frac{1}{\tau_{-1:}^{\rho}} u_{-i}^{\rho[n, m]}-v_{-i}^{\rho[n, m]}\right\|_{a} \\
& \quad=\left\|u_{-j}^{\rho[n, m]}\right\|_{a}\left(\left(\frac{1}{\tau_{-1}^{\rho}}\right)^{2}\left\|u_{-i}^{\rho[n, m]}\right\|_{a}^{2}-\frac{2}{\tau_{-1}^{\rho}} a\left(u_{-i}^{\rho[n, m]}, v_{-i}^{\rho[n, m]}\right)+\left\|v_{-i}^{\rho[n, m]}\right\|_{a}^{2}\right)^{1 / 2} \\
& \quad \rightarrow 0
\end{aligned}
$$


as $n, m \rightarrow \infty$. Due to Theorem 3.4 , the assertion $(1.16)_{b}$ is proved. The proof of assertion (1.16) $)_{a}$ follows the previous pattern.

The equations (4.5) and (4.6) provide the quantitative estimation (1.17), since the norm of the self-adjoint operator $S=(A-\rho I)^{-1} \in \mathcal{L}\left(\mathbf{H}_{a}\right)$ is given by

$$
\|S\|=\max \left\{|\sigma| \in \mathbb{R}^{+}: \sigma \in \sigma(S)\right\}=\frac{1}{\delta^{\rho}} .
$$

Thus the assertion is proved

Proof of Corollary 1.5. According to (2.9) $a$ and $(2.9)_{b}$, we have for $(n, m) \in$ $\mathbb{N} \times \mathbb{N}_{0}$ and $j \in J_{-1}^{\rho}$ always $\left\|v_{-j}^{\rho[n, m]}\right\|_{a} \leq 1$. Due to (4.7), the sequence $\left(P_{-1}^{\rho} u_{-j}^{\rho[n, 0]}\right)$ and the net $\left(P_{-1}^{\rho} u_{-j}^{\rho \mid n, m]}\right)$ are bounded in the finite-dimensional subspace $N_{-1}^{\rho}$. Hence, we have a subsequence $\left(n^{\prime}\right)$ in $\mathbb{N}$ and a subnet $\left(n^{\prime}, m^{\prime}\right)$ in $\mathbb{N} \times \mathbb{N}$, such that by (4.6) the limits $(1.18)_{a}$ and $(1.18)_{b}$ exist, lying in $N_{-1}^{\rho}$. Now by Theorem 1.3 , the system $\left\{\Phi_{-j}^{\rho} \in N_{-1}^{\rho}: j \in J_{-1}^{\rho}\right\}$ constitutes an orthonormal basis of $N_{-1}^{\rho}$

\section{Numerical example I}

We shall consider the eigenvalue problem for partial differential equations given in [13: Section 3]. Let

$$
\Omega=(-1,1) \times(-1,1) \subset \mathbb{R}^{2} \quad \text { and } \quad \mathbf{H}_{a}=H_{0}^{1} \times\left(L_{2}(\Omega) \times L_{2}(\Omega)\right) .
$$

We define

$$
\begin{aligned}
a(u, v)= & \int_{\Omega}\left(\alpha \operatorname{grad} u_{I} \cdot \operatorname{grad} \bar{v}_{I}+\operatorname{grad} u_{I} \cdot \overline{\mathbf{v}}_{I I}+\mathbf{u}_{I I} \cdot \operatorname{grad} \bar{v}_{I}\right. \\
& \left.+u_{I} \bar{v}_{I}+2 \mathbf{u}_{I I} \cdot \overline{\mathbf{v}}_{I I}\right) d \Omega \\
b(u, v)= & \int_{\Omega}\left(u_{I} \bar{v}_{I}+\mathbf{u}_{I I} \cdot \overline{\mathbf{v}}_{I I}\right) d \Omega
\end{aligned}
$$

for $u=\left(u_{I}, \mathbf{u}_{I I}\right)$ and $v=\left(v_{I}, \mathbf{v}_{I I}\right) \in \mathbf{H}_{\alpha}$ with $\alpha \in C^{\infty}(\bar{\Omega}), \alpha(x, y)>0$ and $(2 \alpha-$ 1) $(x, y)>0$ for $(x, y) \in \Omega$.

The inner product $a(\cdot, \cdot)$ gives a norm in $\mathbf{H}_{\mathfrak{a}}$ which is equivalent to the natural norm of this product space. By partial integration one shows that (1.1) is a weak form of the boundary value problem

$$
\begin{aligned}
-\operatorname{div}\left(\alpha \operatorname{grad} u_{I}+\mathbf{u}_{I I}\right) & =(\lambda-1) u_{I} & & \text { in } \Omega \\
\operatorname{grad} u_{I}+\mathbf{u}_{I I} & =(\lambda-1) \mathbf{u}_{I I} & & \text { in } \Omega \\
u_{I} & =0 & & \text { on } \partial \Omega .
\end{aligned}
$$

We set $\mathbf{X}=L_{2}(\Omega) \times\left(L_{2}(\Omega)\right)^{2} \times\left(L_{2}(\Omega)\right)^{2}$,

$$
s(u, v)=\int_{\Omega}\left(\alpha \dot{\mathbf{u}}_{I I I} \cdot \overline{\mathbf{v}}_{I I I}+\mathbf{u}_{I I I} \cdot \overline{\mathbf{v}}_{I I}+\mathbf{u}_{I I} \cdot \overline{\mathbf{v}}_{I I I}+u_{I} \bar{v}_{I}+2 \mathbf{u}_{I I} \cdot \overline{\mathbf{v}}_{I I}\right) d \Omega
$$


for $u=\left(u_{I}, \mathbf{u}_{I I}, \mathbf{u}_{I I I}\right), v=\left(v_{I}, \mathbf{v}_{I I}, \mathbf{v}_{I I I}\right) \in \mathbf{X}$ and

$$
\mathbf{T}: \mathbf{H}_{a} \ni\left(f_{I}, \mathbf{f}_{I I}\right) \longmapsto\left(f_{I}, \mathbf{f}_{I I}, \operatorname{grad} f_{I}\right) \in \mathbf{X} .
$$

In our calculations of bounds we restrict the problem to the symmetry class (invariant subspace)

$$
\mathbf{H}_{a}=H_{0}^{1}(\Omega)^{(0,0)} \times\left(L_{2}(\Omega)^{(1,0)} \times \mathrm{E}_{2}(\Omega)^{(0,1)}\right)
$$

and

$$
\mathbf{X}=L_{2}(\Omega)^{(0,0)} \times\left(L_{2}(\Omega)^{(1,0)} \times L_{2}(\Omega)^{(0,1)}\right) \times\left(L_{2}(\Omega)^{(1,0)} \times L_{2}(\Omega)^{(0,1)}\right),
$$

respectively. We use the same trial functions $v_{1}, \ldots, v_{n} \in \mathbf{H}_{a}$ and $w_{1}^{\circ}, \ldots, w_{m}^{\circ} \in \mathbf{X}^{\circ}$ as in [13: Section 3].

Here, we shall consider $\alpha(x, y)=1$ for $(x, y) \in \Omega$. The corresponding eigenvalue problem can be solved explicitly: $\lambda=1$ and $\lambda=2$ are eigenvalues of infinite multiplicity, forming the essential spectrum. Let $\left(\mu_{k, l}, u_{k, l}\right) \in \mathbb{R} \times H_{0}^{1}(\Omega)$ be the eigenpairs of the Laplacian operator $-\Delta u=\mu u$ with

$$
\begin{aligned}
\mu_{k, l} & =\left(k^{2}+l^{2}\right)\left(\frac{\pi}{2}\right)^{2} \\
u_{k, l}(x, y) & =\sin \left(\frac{k \pi}{2}(x+1)\right) \sin \left(\frac{l \pi}{2}(y+1)\right) \quad((x, y) \in \Omega ; k, l \in \mathbb{N}) .
\end{aligned}
$$

Then the further eigenpairs of (1.1) are given by

$$
\left(2+\mu_{k, l},\left(\mu_{k, l} u_{k, l}, \operatorname{grad} u_{k, l}\right)\right) \in \mathbb{R} \times \mathbf{H}_{a} \quad(k, l \in \mathbb{N})
$$

Thus, we find

$$
\begin{aligned}
2 & <\lambda_{1}=2+\mu_{1,1} \\
& <\lambda_{2} \doteq 2+\mu_{1,3} \\
& =\lambda_{3}=2+\mu_{3,1}<28<30<33<37<40 \\
& <\lambda_{4}=2+\mu_{3,3} \\
& <\lambda_{5}
\end{aligned}
$$

and, with our local notation for $\rho \in\left(\lambda_{3}, \lambda_{4}\right)$,

$$
\begin{aligned}
& N_{-1}^{\rho}=\operatorname{span}\left\{\left(\mu_{1,3} u_{1,3}, \operatorname{grad} u_{1,3}\right),\left(\mu_{3,1} u_{3,1}, \operatorname{grad} u_{3,1}\right)\right\} \subset \mathbf{H}_{a} \\
& N_{+1}^{\rho}=\operatorname{span}\left\{\left(\mu_{3,3} u_{3,3}, \operatorname{grad} u_{3,3}\right)\right\} \subset \mathbf{H}_{a} .
\end{aligned}
$$

See [13: Section 3/Table 1] for upper and lower bounds to the eigenvalues. Now, the results

$$
\begin{aligned}
a\left(\left(\tau_{-i}^{40[108,56]}\right)^{-1} u_{-i}^{40[108,56]},\left(\tau_{-j}^{40[108,56]}\right)^{-1} u_{-j}^{40[108,56]}\right) & \\
= & \begin{cases}1.000000181 & \text { for } i=j=1 \\
1.000000182 & \text { for } i=j=2 \\
1.94 \cdot 10^{-16} & \text { for } i=1, j=2\end{cases}
\end{aligned}
$$


and

$$
a\left(\left(\tau_{+1}^{30[108,56]}\right)^{-1} u_{+1}^{30[108,56]},\left(\tau_{+1}^{30[108,56]}\right)^{-1} u_{+1}^{30[108,56]}\right)=1.00049
$$

put to the test the second limit of $(1.16)_{b}$. The Tables 1 and 2 show the quality of the error estimates of (1.17).

\begin{tabular}{|c|c|c|c|c|}
\hline$j \in J_{-1}^{40}$ & $\left\|\left(I-P_{-1}^{40}\right) u_{-j}^{40[108,56]}\right\|_{a}^{2}$ & $\left(1+\frac{\rho}{\delta^{40}}\right)^{2} \frac{1}{\gamma_{-}^{40}}$ & $\cdot\left[\tau_{-j}^{40[108,56]}-\tau_{-1}^{40}\right.$ & \\
\hline 1 & $5.438 \cdot 10^{-8}$ & 29.23 & $2.625 \cdot 10^{-7}$ & $=7.672 \cdot 10^{-6}$ \\
\hline 2 & $1.037 \cdot 10^{-7}$ & 29.23 & $2.639 \cdot 10^{-7}$ & $=7.713 \cdot 10^{-6}$ \\
\hline
\end{tabular}

Table 1: Approximation of the eigenspace $N_{-1}^{40}$

\begin{tabular}{|c|c|c|c|c|}
\hline$\rho \in\left(\lambda_{3}, \lambda_{1}\right)$ & $\left\|\left(I-P_{+1}^{\rho}\right) u_{+1}^{\rho[108,56]}\right\|_{a}^{2}$ & $\left(1+\frac{\rho}{\delta^{\rho}}\right)^{2} \frac{1}{\gamma_{+}^{\rho}} \cdot[$ & {$\left[\tau_{+1}^{\rho}-\tau_{+1}^{\rho(108,56]}\right]$} & \\
\hline 28 & $3.068 \cdot 10^{-3}$ & 621.8 & $1.365 \cdot 10^{-3}$ & $=8.488 \cdot 10^{-1}$ \\
\hline 30 & $3.915 \cdot 10^{-3}$ & 100.6 & $2.075 \cdot 10^{-3}$ & $=2.088 \cdot 10^{-1}$ \\
\hline $3: 3$ & $5.979 \cdot 10^{-3}$ & 26.38 & $4.196 \cdot 10^{-3}$ & $=1.107 \cdot 10^{-1}$ \\
\hline 37 & $1.244 \cdot 10^{-2}$ & 9.134 & $1.358 \cdot 10^{-2}$ & $=1.239 \cdot 10^{-1}$ \\
\hline
\end{tabular}

Table 2: Approximations of the eigenspace $N_{+1}^{\rho}$ depending on $\rho \in\left(\lambda_{3}, \lambda_{4}\right)$

Mathematica [11] was employed for the symbolic evaluation of all inner products and for the calculation of all matrices. The eigenpairs of (1.6) were calculated by means of a suitable library routine.

\section{Convergence results for the right-definite eigenvalue problem}

Let $\mathbf{H}_{b}$ be a separable, complex Hilbert space with inner product $b(\cdot, \cdot)$ and norm $\|\cdot\|_{b}$. Suppose that $a(\cdot, \cdot)$ is a Hermitian sesquilinear form in $\mathbf{H}_{b}$ with dense domain $\mathbf{H}_{a}$. Furthermore, $a(\cdot, \cdot)$ is assumed to be bounded from below and closed. Without loss of generality, let $a(\cdot, \cdot)$ be an inner product such that for $\kappa>0$

$$
\kappa^{2} b(v, v) \leq a(v, v) \quad\left(v \in \mathbf{H}_{a}\right)
$$

holds. By the closedness of $a(\cdot, \cdot)$, the inner product space $\left(\mathbf{H}_{a}, a(\cdot, \cdot)\right)$ with norm $\|\cdot\|_{a}$ is a Hilbert space (see, e.g., [5: Chapter VI/Subsection 1.3]). We then consider (cf. [13: Section 4]) the following right-definite eigenvalue problem:

$\left.\begin{array}{l}\text { Find eigenpairs }(\lambda, u) \in \mathbb{R} \times \mathbf{H}_{a} \text { with } u \neq 0 \\ \text { such that } a(u, v)=\lambda b(u, v) \text { for all } v \in \mathbf{H}_{a} .\end{array}\right\}$ 
This eigenvalue problem is equivalent to the eigenvalue problem for a self-adjoint and positive definite operator $A$ in $\mathbf{H}_{b}$ defined by

$$
\begin{aligned}
\mathcal{D}(A) & =\left\{u \in \mathbf{H}_{a}: a(\hat{u}, v)=b(\hat{u}, v)\left(v \in \mathbf{H}_{a}\right) \text { for some } \hat{u} \in \mathbf{H}_{b}\right\} \\
b(A u, v) & =a(u, v) \quad \text { for all } u \in \mathcal{D}(A) \text { and } v \in \mathbf{H}_{a}
\end{aligned}
$$

where $\mathcal{D}(A)$ is dense in the Hilbert space $\mathbf{H}_{a}$ (see, e.g., [5: Chapter VI/Subsection 2.1]). Thus, $\|\cdot\|_{a}$ gives the energy norm on $\mathbf{H}_{a}$ performing

$$
\|v\|_{a}^{2}=b(A v, v) .(v \in \mathcal{D}(A)) \quad \text { and } \quad\|v\|_{b} \leq \kappa^{-1}\|v\|_{a} \quad\left(v \in \mathbf{H}_{a}\right) .
$$

Now, let the eigenelements $u_{ \pm i}^{\rho} \in \mathbf{H}_{a}$ of eigenpairs $\left(\lambda_{ \pm i}^{\rho}, u_{ \pm i}^{\rho}\right) \in \mathbb{R} \times \mathbf{H}_{a}$ satisfy

$$
b\left(u_{k}^{\rho}, u_{l}^{\rho}\right)=\delta_{k l} \quad\left(k, l \in\left\{-k_{\rho}^{-}, \ldots,-1,+1, \ldots,+k_{\rho}^{+}\right\}\right)
$$

Again, we assume that $\mathbf{X}$ is a complex Hilbert space with inner product $s(\cdot, \cdot)$, norm $\|\cdot\|_{s}$ and isometry $\mathbf{T}: \mathbf{H}_{b} \longrightarrow \mathbf{X}$,

$$
s(\mathbf{T} u, \mathbf{T} v)=b(u, v) \quad\left(u, v \in \mathbf{H}_{b}\right)
$$

We denote by

$$
\mathbf{X}^{\circ}=\left\{w \in \mathbf{X}: s(w, \mathbf{T} u)=0 \text { for all } u \in \mathbf{H}_{b}\right\} .
$$

the $s(\cdot, \cdot)$-orthogonal complement of $\mathbf{T H}_{b}$ in $\mathbf{X}$. If the eigenvalues $\lambda_{ \pm i}^{\rho}$ are represented in the form

$$
\lambda_{ \pm i}^{\rho}=\rho+\frac{1}{\tau_{ \pm i}^{\rho}} \quad \text { with } \quad \tau_{ \pm i}^{\rho}=\frac{1}{\lambda_{ \pm i}^{\rho}-\rho} \quad\left(i=1, \ldots, k_{\rho}^{ \pm}\right)
$$

then the quantities $\tau_{ \pm i}^{\rho} \in \mathbb{R}$ are characterized again (see [13: Theorem 1.3 and Corollary 4.1]) by variational principles.

For a discretization of the eigenvalue problem (6.1), let $n \in \mathbb{N}$ and $m \in \mathbb{N}_{0}$; and suppose the following:

$(\mathbf{R})_{1} v_{1}, \ldots, v_{n} \in \mathcal{D}(A)$ are linearly independent, $V_{n}=\operatorname{span}\left\{v_{1}, \ldots, v_{n}\right\}, V_{n} \cap D_{\rho}=\{0\}$.

$(\mathbf{R})_{2} w_{1}^{\star}, \ldots, w_{n}^{\star} \in \mathbf{X}$ satisfy $s\left(w_{i}^{\star}, \mathbf{T} u\right)=a\left(v_{i}, u\right)$ for all $u \in \mathbf{H}_{a}(i=1, \ldots, n)$.

(R) $w_{3} w_{0}^{\circ}, \ldots, w_{m}^{\circ} \in \mathbf{X}^{\circ}$ where $w_{0}^{\circ}=0$ and $w_{1}^{\circ}, \ldots, w_{m}^{\circ}$ are linearly independent.

Then, we construct matrices $A_{0} \doteq\left(a_{i k}^{(0)}\right), A_{1}=\left(a_{i k}^{(1)}\right), A_{2}=\left(a_{i k}^{(2)}\right)$ by

$$
\begin{aligned}
& a_{i k}^{(0)}=b\left(A v_{k}, A v_{i}\right) \\
& a_{i k}^{(1)}=a\left(v_{k}, v_{i}\right) \\
& a_{i k}^{(2)}=b\left(v_{k}, v_{i}\right)
\end{aligned} \quad \quad(1 \leq i, k \leq n), \quad . \quad
$$


and matrices $B_{11}=\left(b_{i k}^{(11)}\right), B_{12}=\left(b_{i k}^{(12)}\right), B_{22}=\left(b_{i k}^{(22)}\right)$ by

$$
\begin{array}{ll}
b_{i k}^{(11)}=s\left(w_{k}^{*}, w_{i}^{*}\right) & (1 \leq i, k \leq n) \\
b_{i k}^{(12)}=\left\{\begin{array}{lll}
s\left(w_{k}^{\circ}, w_{i}^{*}\right) & \text { for } m>0 \\
0 & \text { for } m=0
\end{array}\right. & (1 \leq i \leq n ; 1 \leq k \leq \max \{1, m\}) \\
b_{i k}^{(22)}=\left\{\begin{array}{ll}
s\left(w_{k}^{\circ}, w_{i}^{\circ}\right) & \text { for } m>0 \\
1 & \text { for } m=0
\end{array} \quad(1 \leq i, k \leq \max \{1, m\}),\right.
\end{array}
$$

and consider the matrix eigenvalue problem

$$
\left(A_{1}-\rho A_{2}\right) x=\tau\left(B_{11}-B_{12} B_{22}^{-1} B_{12}^{H}-2 \rho A_{1}+\rho^{2} A_{2}\right) x
$$

where $\tau \in \mathbb{R}$ and $x \in \mathbb{C}^{n}$. Denote all non-zero eigenvalues of (6.2) by $\tau_{ \pm i}^{\rho[n, m]}$ and arrange them in the order

$$
\tau_{-1}^{\rho[n, m]} \leq \cdots \leq \tau_{-n^{-}}^{\rho[n, m]}<0<\tau_{+n^{+}}^{\rho[n, m]} \leq \cdots \leq \tau_{+1}^{\rho[n, m]}
$$

where $n^{-}, n^{+} \in \mathbb{N}_{0}$ with $n^{-}+n^{+} \leq n$. Then we get (see [13: Theorem 4.2]) the eigenvalue bounds

$$
\Lambda_{-i}^{\rho[n, m]}=\rho+\left(\tau_{-i}^{\rho[n, m]}\right)^{-1} \leq \lambda_{-i}^{\rho} \quad\left(i=1, \ldots, \min \left\{n^{-}, k_{\rho}^{-}\right\}\right)
$$

and

$$
\Lambda_{+i}^{\rho[n, m]}=\rho+\left(\tau_{+i}^{\rho[n, m]}\right)^{-1} \geq \lambda_{+i}^{\rho} \quad\left(i=1, \ldots, \min \left\{n^{+}, k_{\rho}^{+}\right\}\right) .
$$

Assume the eigenvectors of (6.2) to form an orthonormal system with respect to the inner product

$$
\mathbb{C}^{n} \times \mathbb{C}^{n} \ni(x, y) \longmapsto x^{H}\left(B_{11}-B_{12} B_{22}^{-1} B_{12}^{H}-2 \rho A_{1}+\rho^{2} A_{2}\right) y \in \mathbb{C} .
$$

Now, if $\left(\tau_{ \pm i}^{\rho[n, m]}, x\right) \in \mathbb{R} \times \mathbb{C}^{n}$ with $x=\left(x_{1}, \ldots, x_{n}\right)^{\ell} \in \mathbb{C}^{n}$ is an eigenpair of (6.2), we set

$$
u_{ \pm i}^{\rho[n, m]}=\sum_{j=1}^{n} x_{j} v_{j} \in V_{n} \quad\left(i=1, \ldots, n^{ \pm}\right)
$$

This orthonormalization is equivalent to

$$
b\left((A-\rho I) u_{k}^{\rho[n, m]}, u_{l}^{\rho[n, m]}\right)=\delta_{k l} \tau_{k}^{\rho[n, m]}\left(k, l \in\left\{-1, \ldots,-n^{-},+n^{+}, \ldots,+1\right\}\right) .
$$

Remark 6.1. Observe that the quantities (6.3) - (6.6) are denoted exactly in the same manner as the corresponding ones in Section 1.

Now, assume (1.11) to be fulfilled, and

$$
\bigcup_{n \in \mathbb{N}}(A-\rho I)\left(V_{n}\right) \text { is dense in }\left(\mathbf{H}_{b},\|\cdot\|_{b}\right) \text {. }
$$

Theorem 6.2. Let $0<\rho \notin \sigma(A)$. Then the assertions of Theorem 1.2 hold.

Denote by

$$
P_{ \pm 1}^{\rho}: \mathrm{H}_{\delta} \longrightarrow N_{ \pm 1}^{\rho}
$$

the $b(\cdot, \cdot)$-orthogonal projection of $\mathbf{H}_{b}$ onto the eigenspace $N_{ \pm 1}^{\rho}$. Furthermore, we adapt from (1.14) and (1.15) the definition of $\delta^{\rho}, \gamma_{-}^{\rho}$ and $\gamma_{+}^{\rho}$. 
Theorem 6.3. Let $0<\rho \notin \sigma(A)$. Then:

For $i, j \in J_{ \pm 1}^{\rho}$ we have the limits

$$
\left.\begin{array}{r}
\lim _{n \rightarrow \infty}\left\|\left(I-P_{ \pm 1}^{\rho}\right) u_{ \pm i}^{\rho[n, 0]}\right\|_{a}=0 \\
\lim _{n \rightarrow \infty} b\left(\left(\tau_{ \pm i}^{\rho[n, 0]}\right)^{-1} u_{ \pm i}^{\rho[n, 0]},\left(\tau_{ \pm j}^{\rho[n, 0]}\right)^{-1} u_{ \pm j}^{\rho[n, 0]}\right)=\delta_{i j}
\end{array}\right\}
$$

and

$$
\left.\begin{array}{c}
\lim _{n, m \rightarrow \infty}\left\|\left(I-P_{ \pm 1}^{\rho}\right) u_{ \pm i}^{\rho[n, m]}\right\|_{a}=0 \\
\lim _{n, m \rightarrow \infty} b\left(\left(\tau_{ \pm i}^{\rho[n, m]}\right)^{-1} u_{ \pm i}^{\rho[n, m]},\left(\tau_{ \pm j}^{\rho[n, m]}\right)^{-1} u_{ \pm j}^{\rho[n, m]}\right)=\delta_{i j} .
\end{array}\right\}
$$

For $m \in \mathbb{N}_{0}$ and sufficiently large $n \in \mathbb{N}$ we have the quantitative estimates

$$
\left\|\left(I-P_{-1}^{\rho}\right) u_{-j}^{\rho[n, m]}\right\|_{a}^{2} \leq\left(1+\frac{\rho}{\delta^{\rho}}\right) \frac{1}{\delta^{\rho} \gamma_{-}^{\rho}}\left[\tau_{-j}^{\rho[n, m]}-\tau_{-1}^{\rho}\right] \quad\left(j \in J_{-1}^{\rho}\right)
$$

and

$$
\left\|\left(I-P_{+1}^{\rho}\right) u_{+j}^{\rho[n, m]}\right\|_{a}^{2} \leq\left(1+\frac{\rho}{\delta^{\rho}}\right) \frac{1}{\delta^{\rho} \gamma_{+}^{\rho}}\left[\tau_{+1}^{\rho}-\tau_{+j}^{\rho[n, m]}\right] \quad\left(j \in J_{+1}^{\rho}\right) .
$$

Corollary 6.4. There exists a subsequence $\left(n^{\prime}\right)$ in $\mathbb{N}$, a subnet $\left(n^{\prime}, m^{\prime}\right)$ in $\mathbb{N} \times \mathbb{N}$ and a $b(\cdot, \cdot)$-orthonormal basis

$$
\left\{\Phi_{ \pm j}^{\rho} \in N_{ \pm 1}^{\rho}: j \in J_{ \pm 1}^{\rho}\right\}
$$

of $N_{ \pm 1}^{\rho}$ such that for $j \in J_{ \pm 1}^{\rho}$ the limits $(1.18)_{a}$ and $(1.18)_{b}$ hold (with respect to the energy norm on $\mathbf{H}_{\mathbf{a}}$ ).

In the following section we sketch the proofs of the Theorems 6.2 and 6.3 giving mainly the differences to the left-definite case.

\section{Proofs for the right-definite case}

Due to our assumptions, we have $\rho \notin \sigma(A)$. In particular,

$$
T=(A-\rho I)^{-1} \in \mathcal{L}\left(\mathbf{H}_{b}\right)
$$

is a bounded and self-adjoint operator. Obviously, $\left(\lambda_{-i}^{\rho}, u_{-i}^{\rho}\right) \in \mathbb{R} \times \mathbf{H}_{a}$ is an eigenpair of (6.1) if and only if $\left(\tau_{-i}^{\rho}, u_{-i}^{\rho}\right) \in \mathbb{R} \times \mathbf{H}_{a}$ is an eigenpair of $T \in \mathcal{L}\left(\mathbf{H}_{b}\right)$ with $\tau_{-i}^{\rho}=\frac{1}{\lambda_{-i}^{\rho}-\rho}$ for $i \in\left\{1, \ldots, k_{\rho}^{-}\right\}$. We set

$$
W_{n}=(A-\rho I)\left(V_{n}\right) \subset \mathbf{H}_{b} \quad(n \in \mathbb{N}) .
$$

Due to (6.8),

$$
\bigcup_{n \in N} W_{n} \quad \text { is dense in }\left(\mathbf{H}_{b},\|\cdot\|_{b}\right)
$$


Based on the assumptions $(\mathrm{R})_{1}-(\mathrm{R})_{3}$, we define for $(n, m) \in \mathbb{N} \times \mathbb{N}_{0}$ the Goerisch mapping $G_{m}: V_{n} \longrightarrow \mathrm{X}$ by

$$
G_{m}(v)= \begin{cases}\mathrm{T} A v & \text { for } m=0 \\ w^{\star}-Q_{m}^{\circ} w^{\star} & \text { for } m \geq 1\end{cases}
$$

with

$$
v=\sum_{j=1}^{n} x_{j} v_{j} \in V_{n} \quad \text { and } \quad w^{\star}=\sum_{j=1}^{n} x_{j} w_{j}^{\star} \in \mathbf{X}
$$

where $x=\left(x_{1}, \ldots, x_{n}\right)^{t} \in \mathbb{C}^{n}$. Then we have

$$
\begin{aligned}
x^{H}\left(B_{11}-B_{12} B_{22}^{-1} B_{12}^{H}\right) x & =\min \left\{s\left(w^{\star}+w, w^{\star}+w\right): w \in \mathbf{X}_{m}^{\circ}\right\} \\
& =s\left(G_{m} v, G_{m} v\right) \\
& \geq x^{H} A_{0} x=b(A v, A v)=s(\mathbf{T} A v, \mathbf{T} A v) .
\end{aligned}
$$

For the $(n \times n)$-matrix $A_{0, m}=B_{11}-B_{12} B_{22}^{-1} B_{12}^{H}$, the limit

$$
x^{H}\left(A_{0, m}-A_{0}\right) x=\left\|G_{m} v-\mathbf{T} A v\right\|_{s}^{2} \downarrow 0
$$

as $m \rightarrow \infty$ holds. Furthermore for $(n, m) \in \mathbb{N} \times \mathbb{N}_{0}$ and $i \in\left\{1, \ldots, n^{-}\right\}$we have

$$
\begin{aligned}
\left\|(A-\rho I) u_{-i}^{\rho[n, m]}\right\|_{b}^{2}+\left\|\left(G_{m}-\mathbf{T} A\right) u_{-i}^{\rho[n, m]}\right\|_{b}^{2} & =1 \\
\lim _{m \rightarrow \infty}\left\|(A-\rho I) u_{-i}^{\rho[n, m]}\right\|_{b} & =1 .
\end{aligned}
$$

For $n \in \mathbb{N}, x=\left(x_{1}, \ldots, x_{n}\right)^{t} \in \mathbb{C}^{n}$ and $v=\sum_{j=1}^{n} x_{j} v_{j} \in V_{n}$, the Rayleigh quotient of $(6.2)$ is given by

$$
T_{R, m}^{\rho}(v)=\frac{b((A-\rho I) v, v)}{\|(A-\rho I) v\|_{b}^{2}+\left\|\left(G_{m}-\mathrm{T} A\right) v\right\|_{s}^{2}} \quad\left(m \in \mathbb{N}_{0}\right) .
$$

If we introduce the notation (2.13) for the eigenvalues of (6.2), Poincarés principle yields

$$
\tilde{\tau}_{i}^{\rho[n, m]}=\min _{\substack{v \subset v_{n} \\ d i m v=i}} \max _{\substack{0 \neq v \in V \\ R, m}} T_{R, m}^{\rho}(v) \quad(i=1, \ldots, n) .
$$

In the special case $m=0$ (Lehmann-Maehly method) we substitute $v=T w$ and obtain from (7.1)

$$
\tilde{\tau}_{i}^{\rho[n, 0]}=\min _{\substack{W \mathcal{C}_{n} \\ \text { dimw } w=i}} \max _{0 \neq w \in W} \frac{b(T w, w)}{b(w, w)} \quad(i=1, \ldots, n) .
$$

Theorem 7.1. For $i \in\left\{1, \ldots, k_{\rho}^{-}\right\}$and $n \in \mathbb{N}$ with $n \geq k_{\rho}^{-}$, we have

$$
\tau_{-i}^{\rho} \leq \tilde{\tau}_{i}^{\rho[n, 0]} .
$$

Moreover, the quantities $\tilde{\tau}_{i}^{\rho[n, 0]} \in \mathbb{R}$ are upper Rayleigh-Ritz bounds to the eigenvalues $\tau_{-i}^{\rho}$ of the operator $T \in \mathcal{L}\left(\mathbf{H}_{b}\right)$ with respect to the trial functions $(A-\rho I) v_{1}, \ldots,(A-$ $\rho I) v_{n} \in \mathbf{H}_{b}$.

Now, the lemmata and the theorem of Section 3 hold analogously. 
Proof of Theorem 6.2. By the transformation $\tau \cdot \longmapsto \rho+\frac{1}{\tau}$, Theorem 3.4 provides the assertion

For $v \in \mathbf{H}_{b}, u \in \mathcal{D}(A)$ and $v=(A-\rho I) u \neq 0$ we have

$$
\left\|\left(I-P_{-1}^{\rho}\right) v\right\|_{b}^{2} \leq \frac{b(v, v)}{\gamma_{-}^{\rho}}\left[T_{R, 0}^{\rho}(u)-\tau_{-1}^{\rho}\right] .
$$

Proof of Theorem 6.3. For $m \in \mathbb{N}_{0}$ and sufficiently large $n \in \mathbb{N}$ we set

$$
v_{-j}^{\rho[n, m]}=(A-\rho I) \dot{u}_{-j}^{\rho[n, m]} \quad\left(j \in J_{-1}^{\rho}\right)
$$

giving

$$
b\left(v_{-j}^{\rho[n, m]}, v_{-j}^{\rho[n, m]}\right) \leq 1 \quad\left(j \in J_{-1}^{\rho}\right)
$$

and

$$
0>\tau_{-j}^{\rho[n, m]}=T_{R, m}^{\rho}\left(u_{-j}^{\rho[n, m]}\right) \geq T_{R, 0}^{\rho}\left(u_{-j}^{\rho[n, m]}\right) .
$$

For $j \in J_{-1}^{\rho}$ estimation (7.2) yields

$$
\left\|\left(I-P_{-1}^{\rho}\right) v_{-j}^{\rho[n, m]}\right\|_{b}^{2} \leq \frac{1}{\gamma_{-}^{\rho}}\left[\tau_{-j}^{\rho[n, m]}-\tau_{-1}^{\rho}\right]
$$

and consequently

$$
\begin{array}{r}
\lim _{n \rightarrow \infty}\left\|\left(I-P_{-1}^{\rho}\right) v_{-j}^{\rho[n, 0]}\right\|_{b}=0 \\
\lim _{n, m \rightarrow \infty}\left\|\left(I-P_{-1}^{\rho}\right) v_{-j}^{\rho[n, m]}\right\|_{b}=0 .
\end{array}
$$

For $j \in J_{-1}^{\rho}$ the limit $(7.3)_{b}$ provides

$$
\left(I-P_{-1}^{\rho}\right) u_{-j}^{\rho[n, m]}=(A-\rho I)^{-1}\left(I-P_{-1}^{\rho}\right) v_{-j}^{\rho[n, m]} \longrightarrow 0
$$

as $n, m \rightarrow \infty$, furthermore

$$
A\left(I-P_{-1}^{\rho}\right) u_{-j}^{\rho[n, m]}=\left(I-P_{-1}^{\rho}\right) v_{-j}^{\rho[n, m]}+\rho\left(I-P_{-1}^{\rho}\right) u_{-j}^{\rho[n, m]} \longrightarrow 0
$$

as $n, m \rightarrow \infty$ and finally

$$
\left\|\left(I-P_{-1}^{\rho}\right) u_{-j}^{\rho[n, m]}\right\|_{a} \longrightarrow 0
$$

as $n, m \rightarrow \infty$. Based on

$$
\begin{aligned}
\left(\lambda_{-1}^{\rho}-\rho\right)^{2}\left\|P_{-1}^{\rho} u_{-j}^{\rho[n, m]}\right\|_{b}^{2} & =\left\|(A-\rho I) P_{-1}^{\rho} u_{-j}^{\rho[n, m]}\right\|_{b}^{2} \\
& =\left\|P_{-1}^{\rho} v_{-j}^{\rho[n, m]}\right\|_{b}^{2} \\
& =\left\|v_{-j}^{\rho[n, m]}\right\|_{b}^{2}-\left\|\left(I-P_{-1}^{\rho}\right) v_{-j}^{\rho[n, m]}\right\|_{b}^{2} \\
& \rightarrow 1
\end{aligned}
$$


as $n, m \rightarrow \infty$, the limits $(6.9)_{a}$ and $(6.9)_{b}$ can be proved in the same manner as in Section 4 .

The quantitative estimate (6.10) follows from the equations (7.4) and (7.5) by

$$
\begin{aligned}
\|(I- & \left.P_{-1}^{\rho}\right) u_{-j}^{\rho[n, m]} \|_{a}^{2} \\
& =b\left(A\left(I-P_{-1}^{\rho}\right) u_{-j}^{\rho[n, m]},\left(I-P_{-1}^{\rho}\right) u_{-j}^{\rho[n, m]}\right) \\
& \leq\left(\left\|\left(I-P_{-1}^{\rho}\right) v_{-j}^{\rho[n, m]}\right\|_{b}+\frac{\rho}{\delta^{\rho}}\left\|\left(I-P_{-1}^{\rho}\right) v_{-j}^{\rho[n, m]}\right\|_{b}\right) \frac{1}{\delta^{\rho}}\left\|\left(I-P_{-1}^{\rho}\right) v_{-j}^{\rho[n, m]}\right\|_{b} \\
& =\left(1+\frac{\rho}{\delta^{\rho}}\right) \frac{1}{\delta^{\rho}}\left\|\left(I-P_{-1}^{\rho}\right) v_{-j}^{\rho[n, m]}\right\|_{b}^{2} \\
& \cdots\left(1+\frac{\rho}{\delta^{\rho}}\right) \frac{1}{\gamma_{-}^{\rho} \delta^{\rho}}\left[\tau_{-j}^{\rho[n, m]}-\tau_{-1}^{\rho}\right] \quad\left(j \in J_{-1}^{\rho}\right)
\end{aligned}
$$

and the assertions are proved

Remark 7.2. Theorem 7.1 gives reasons for the rigorous demand (6.8) on the trial functions. In return; Theorem 6.3 yields convergence with respect to the energy norm.

\section{Numerical example II}

Here, we shall consider the eigenvalue problem of Section 5 for $\alpha(x, y)=1+x^{2} y^{2}((x, y)$ $\in \Omega)$, now given on the domain $\Omega=(-a, a) \times(-b, b) \subset \mathbb{R}^{2}$ with $a b=1$. In this case, there exists the essential spectrum $\sigma_{e} \subset(0,2]$ and the point spectrum in $(2,+\infty)$ whereas $\lambda=2=\max \sigma_{e}$ is an isolated eigenvalue of infinite multiplicity. We shall regard the side length ratio $s=\frac{a}{b}$ as a system parameter.

We use polynomial trial functions $v_{1}, \ldots, v_{n} \in \mathcal{D}(A) \subset \mathbf{H}_{b}$, orthogonalized with respect to the inner product $b(\cdot, \cdot)$ and generated via a suitable enumeration by the functions

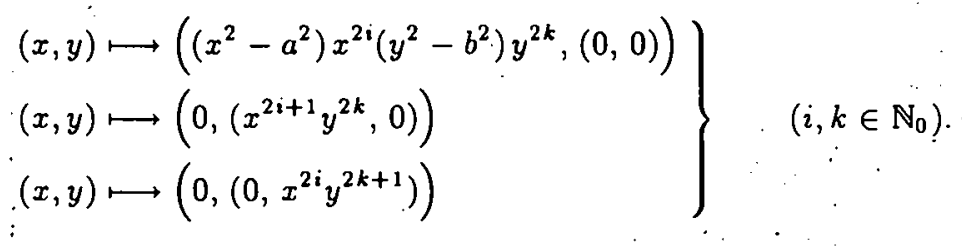

Since the operator $A$ in $\mathbf{H}_{b}$ is well known, the right-definite Lehmann-Maehly method can be applied with these trial functions (cf. [13: Section 4]). Thus, by (6.4) and (6.5) we get upper and lower bounds to the eigenvalues depending on the parameter $s$. The Tables 3 - 5 give bounds for $s=1.1, s=1.53$ and $s=1.9$, respectively. 


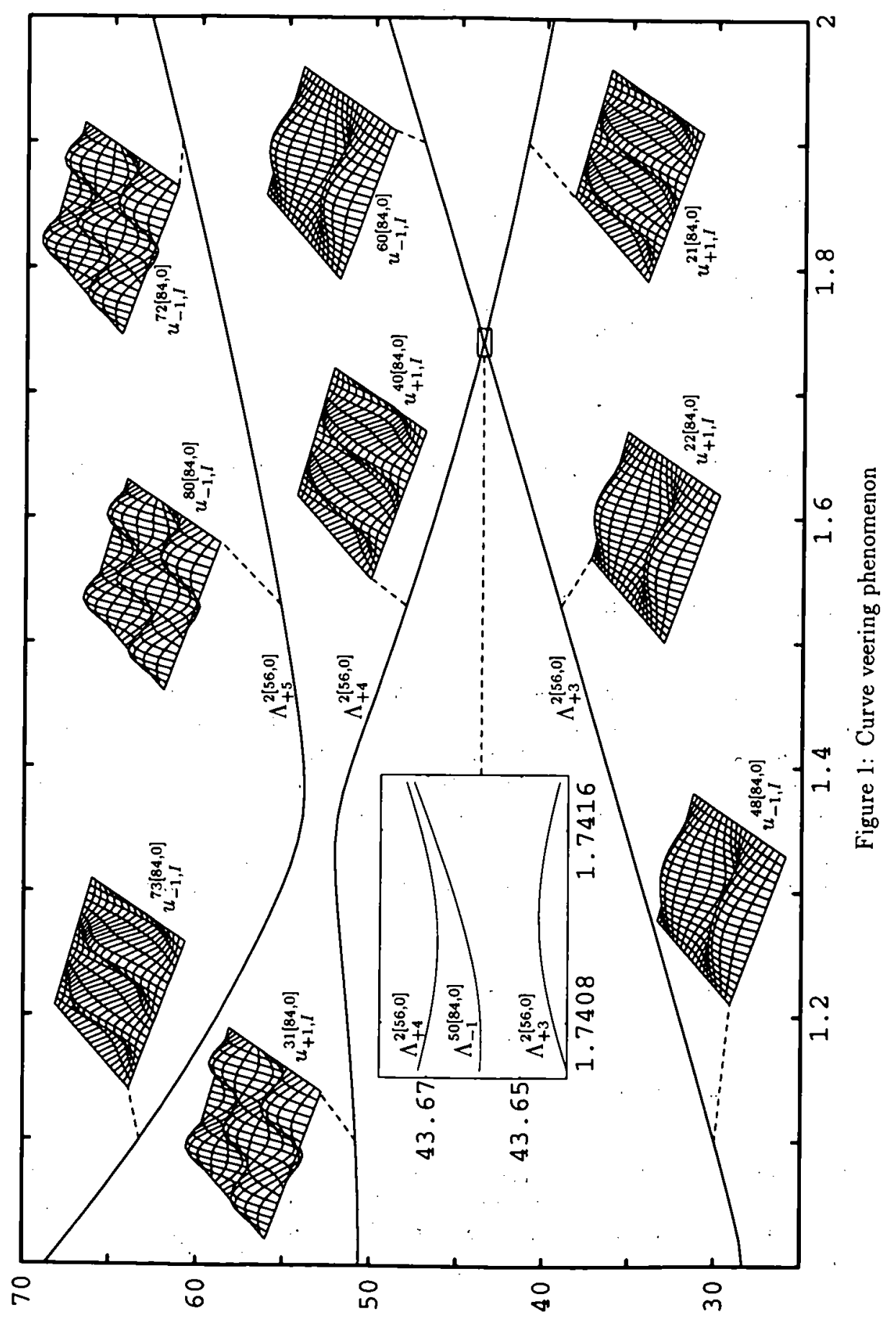




\begin{tabular}{|c||c|c|c|c|c|c|c|c|}
\hline \multicolumn{10}{|c|}{ Upper and lower bounds to the eigenvalues for $s=1.1$} \\
\hline$\rho$ & $\lambda_{1}$ & $\lambda_{2}$ & $\lambda_{3}$ & $\lambda_{4}$ & $\lambda_{5}$ & $\lambda_{6}$ & $\lambda_{7}$ & $\lambda_{8}$ \\
\hline \hline 2 & $\Lambda_{+1}^{\rho}=$ & $\Lambda_{+2}^{\rho}=$ & $\Lambda_{+3}^{\rho}=$ & $\Lambda_{+4}^{\rho}=$ & $\Lambda_{+5}^{\rho}=$ & $\Lambda_{+6}^{\rho}=$ & $\Lambda_{+7}^{\rho}=$ & $\Lambda_{+8}^{\rho}=$ \\
& 7.230459699 & 26.036676 & 29.974332 & 50.8020 & 63.2713 & 74.7699 & 89.745 & 99.326 \\
\hline 110 & $\begin{array}{c}\Lambda_{-8}^{\rho}= \\
7.230459663\end{array}$ & $\Lambda_{-7}^{\rho}=$ & $\Lambda_{-6}^{\rho}=$ & $\Lambda_{-5}^{\rho}=$ & $\Lambda_{-4}^{\rho}=$ & $\Lambda_{-3}^{\rho}=$ & $\Lambda_{-2}^{\rho}=$ & $\Lambda_{-1}^{\rho}=$ \\
& 26.036662 & 29.974316 & 50.7991 & 63.2668 & 74.7641 & 88.973 & 96.656 \\
\hline
\end{tabular}

Table 3: $\Lambda_{+i}^{\rho}=\Lambda_{+i}^{2[56,0]}$ and $\Lambda_{-i}^{\rho}=\Lambda_{-i}^{110[84,0]} ; \Lambda_{+9}^{2[56,0]}=119.33$

\begin{tabular}{|c||c|c|c|c|c|c|c|c|}
\hline \multicolumn{10}{|c|}{ Upper and lower bounds to the eigenvalues for $s=1.53$} \\
\hline$\rho$ & $\lambda_{1}$ & $\lambda_{2}$ & $\lambda_{3}$ & $\lambda_{4}$ & $\lambda_{5}$ & $\lambda_{6}$ & $\lambda_{-}$ & $\lambda_{x}$ \\
\hline \hline 2 & $\Lambda_{+1}^{\rho}=$ & $\Lambda_{+2}^{\rho}=$ & $\Lambda_{+3}^{\rho}=$ & $\Lambda_{+4}^{\rho}=$ & $\Lambda_{+5}^{\rho}=$ & $\Lambda_{+6}^{\rho}=$ & $\Lambda_{+7}^{\rho}=$ & $\Lambda_{+8}^{\rho}=$ \\
& 7.6864688682 & 21.400952 & 39.065869 & 48.0362 & 55.3017 & 83.0767 & 89.119 & 101.08 \\
\hline 110 & $\begin{array}{c}\Lambda_{-8}^{\rho}= \\
7.686468637\end{array}$ & $\Lambda_{-\tau}^{\rho}=$ & $\Lambda_{-6}^{\rho}=$ & $\Lambda_{-5}^{\rho}=$ & $\Lambda_{-4}^{\rho}=$ & $\Lambda_{-3}^{\rho}=$ & $\Lambda_{-2}^{\rho}=$ & $\Lambda_{-1}^{\rho}=$ \\
& 21.400942 & 39.065838 & 48.0328 & 55.2988 & 82.6021 & 87.778 & 101.04 \\
\hline
\end{tabular}

Table 4: $\Lambda_{+i}^{\rho}=\Lambda_{+i}^{2[56,0]}$ and $\Lambda_{-i}^{\rho}=\Lambda_{-i}^{110[84,0]} ; \Lambda_{+9}^{2[56,0]}=121.8$

\begin{tabular}{|c|c|c|c|c|c|c|c|}
\hline \multicolumn{8}{|c|}{ Upper and lower bounds to the eigenvalues for $s=1.9$} \\
\hline$\rho$ & $\lambda_{1}$ & $\lambda_{2}$ & $\lambda_{3}$ & $\lambda_{4}$ & $\lambda_{5}$ & $\lambda_{6}$ & $\lambda_{7}$ \\
\hline 2 & $\begin{array}{c}\Lambda_{+1}^{n}= \\
8.31995553444\end{array}$ & $\begin{array}{c}\Lambda_{+2}^{\rho}= \\
19.51413 .5\end{array}$ & $\begin{array}{c}\Lambda_{+3}^{p}= \\
41.06 .3992\end{array}$ & $\begin{array}{c}\Lambda_{+4}^{\rho}= \\
47.1348\end{array}$ & $\begin{array}{c}=\Lambda_{+5}^{\rho}= \\
61.1291\end{array}$ & $\begin{array}{c}\Lambda_{+6}^{\rho}= \\
73.4526\end{array}$ & $\begin{array}{l}\Lambda_{+7}^{\rho}= \\
84.45 .3\end{array}$ \\
\hline 110 & $\begin{array}{c}\Lambda_{-i}^{n}= \\
8.310955 .52 \times 5)\end{array}$ & $\begin{array}{c}\Lambda_{-6}^{\rho}= \\
19.514126\end{array}$ & $\begin{array}{c}\Lambda_{-5}^{\rho}= \\
41.0613466\end{array}$ & $\begin{array}{c}\Lambda_{-4}^{\prime \prime}= \\
47.1: 346\end{array}$ & $\begin{array}{c}\Lambda_{-3}^{\rho}= \\
61.1254\end{array}$ & $\begin{array}{c}\Lambda_{-2}^{\rho}= \\
73.0028\end{array}$ & $\begin{array}{l}\Lambda_{-1}^{\rho}= \\
83.834\end{array}$ \\
\hline
\end{tabular}

Table 5: $\Lambda_{+i}^{\rho}=\Lambda_{+i}^{2[56,0]}$ and $\Lambda_{-i}^{\rho}=\Lambda_{-i}^{110[84,0]} ; \Lambda_{+8}^{2[56,0]}=123.1$

When plotting the approximations of eigenvalues $\lambda_{3}, \lambda_{4}$ and $\lambda_{5}$ versus the side length ratio $s \in[1,2]$, the famous curve veering phenomenon can be observed (see Figure 1). According to Theorem 6.3, the graphs of the first component of the eigenelement approximations $u_{ \pm i}^{\rho[n, m]} \in \mathbf{H}_{b}$ are plotted in Figure 1 for the indicated values of $s$ and the spectral parameters $\rho$ chosen appropriately with respect to the Tables $3-5$.

So far, this phenomenon has been described only for eigenvalues below the essential spectrum (see, e.g., $[1,2]$ and the references given there). 


\section{References}

[1] Behnke, H.: A numerically rigorous proof of curve veering in an eigenvalue problem for differential equations. Z. Anal. Anw. 15 (1996), $181-200$.

[2] Behnke, H. and U. Mertins: Eigenwertschranken für das Problem der freischwingenden rechteckigen Platte und Untersuchungen zum Ausweichphänomen. Z. Angew. Math. Mech. (ZAMM) 75 (1995), 343 - 363.

[3] Goerisch, F.: Eine Verallgemeinerung eines Verfahrens von N. J. Lehmann zur Einschließung von Eigenwerten. Wiss. Z. Tech. Univ. Dresden 29 (1980), $429-431$.

[4] Goerisch, F. and J. Albrecht: The convergence of a new method for calculating lower bounds to eigenvalues. Lec. Notes Math. 1192 (1986), $303-308$.

[5] Kato,T.: Perturbation Theory for Linear Operators. 2nd Ed. Berlin - Heidelberg - New - York: Springer-Verlag 1976.

[6] Lehmann, N. J.: Beiträge zur numerischen Lösung linearer Eigenwertprobleme. Parts I und II. Z. Angew. Math. Mech. (ZAMM) 29 (1949), $341-356$ and 30 (1950), $1-16$.

[7] Maehly, H. J.: Ein neues Verfahren zur genäherten Berechnung der Eigenwerte hermitescher Operatoren. Helv. Phys. Acta 25 (1952), $547-568$.

[8] Mertins, U.: Zur Konvergenz des Rayleigh-Ritz-Verfahrens bei Eigenwertaufgaben. Numer. Math. 59 (1991), 667 - 682.

[9] Mertins, U.: Asymptotische Fehlerschranken für Rayleigh-Ritz-Approximationen selbstadjungierter Eigenwertaufgaben. Numer. Math. 63 (1992), 227-. 241.

[10] Mertins, U.: Schranken für Eigenwerte bei Aufgaben mit Differentialgleichungen - Verfahren und ihre Konvergenz. Z. Angew. Math. Mech. (ZAMM) 75/SII (1995), S413 S416.

[11] Wolfram, S.: Mathematica. A System for Doing Mathematics by Computer. 2nd Ed. Redwood City (California): Addison-Wesley 1991.

[12] Zimmermann, S.: Comparison of errors in upper and lower bounds to eigenvalues of selfadjoint eigenvalue problems. Numer. Funct. Anal. Optim. 15 (1994), 943 - 960.

[13] Zimmermann, S. and U. Mertins: Variational bounds to eigenvalues of self-adjoint eigenvalue problems with arbitrary spectrum. Z. Anal. Anw. 14 (1995), $327-345$. 